TRANSACTIONS OF THE

AMERICAN MATHEMATICAL SOCIETY

Volume 358, Number 1, Pages 329-358

S 0002-9947(05)03712-8

Article electronically published on March 31, 2005

\title{
THE AUTOMORPHISM TOWER OF GROUPS ACTING ON ROOTED TREES
}

\author{
LAURENT BARTHOLDI AND SAID N. SIDKI
}

\begin{abstract}
The group of isometries $\operatorname{Aut}\left(\mathcal{T}_{n}\right)$ of a rooted $n$-ary tree, and many of its subgroups with branching structure, have groups of automorphisms induced by conjugation in $\operatorname{Aut}\left(\mathcal{T}_{n}\right)$. This fact has stimulated the computation of the group of automorphisms of such well-known examples as the group $\mathfrak{G}$ studied by R. Grigorchuk, and the group $\ddot{\Gamma}$ studied by N. Gupta and the second author.

In this paper, we pursue the larger theme of towers of automorphisms of groups of tree isometries such as $\mathfrak{G}$ and $\ddot{\Gamma}$. We describe this tower for all subgroups of $\operatorname{Aut}\left(\mathcal{T}_{2}\right)$ which decompose as infinitely iterated wreath products. Furthermore, we fully describe the towers of $\mathfrak{G}$ and $\ddot{\Gamma}$.

More precisely, the tower of $\mathfrak{G}$ is infinite countable, and the terms of the tower are 2-groups. Quotients of successive terms are infinite elementary abelian 2-groups.

In contrast, the tower of $\ddot{\Gamma}$ has length 2 , and its terms are $\{2,3\}$-groups. We show that $\operatorname{Aut}^{2}(\ddot{\Gamma}) / \operatorname{Aut}(\ddot{\Gamma})$ is an elementary abelian 3-group of countably infinite rank, while $\operatorname{Aut}^{3}(\ddot{\Gamma})=\operatorname{Aut}^{2}(\ddot{\Gamma})$.
\end{abstract}

\section{INTRODUCTION}

The completeness of a centerless 11 group is measured by the extent to which all its automorphisms are inner, i.e. to which $G=$ Aut $G$. If $G$ has outer automorphisms, then adjoining them to $G$ is part of the process of completing $G$; indeed $G$ embeds in Aut $G$ as the subgroup of inner automorphisms, and Aut $G$ is the one-step completion of $G$. This process can be repeated on Aut $G$ to produce a subnormal series of automorphism groups Aut $^{i} G$ for all $i \in \mathbb{N}$. Formally, the automorphism tower of $G$ is defined as follows:

Definition 1.1. Given a centerless group $G$, we define $\operatorname{Aut}^{\alpha}(G)$ for any ordinal $\alpha$ as follows:

$$
\begin{gathered}
\operatorname{Aut}^{0}(G)=G ; \\
\operatorname{Aut}^{\alpha+1}(G)=\operatorname{Aut}\left(\operatorname{Aut}^{\alpha}(G)\right) ;
\end{gathered}
$$

Received by the editors August 15, 2003 and, in revised form, March 12, 2004.

2000 Mathematics Subject Classification. Primary 20F28; Secondary 20E08.

The authors gratefully acknowledge support from the "Fonds National Suisse de la Recherche Scientifique".

${ }^{1}$ In the sense that $G$ has a trivial center.

(C)2005 American Mathematical Society Reverts to public domain 28 years from publication 
and if $\alpha$ is a limit ordinal, then

$$
\operatorname{Aut}^{\alpha}(G)=\bigcup_{\beta<\alpha} \operatorname{Aut}^{\beta}(G) .
$$

All these groups are also centerless, and hence form an ascending tower of groups.

We define the height $\tau(G)$ as the least ordinal $\alpha$ such that $\operatorname{Aut}^{\alpha}(G)=\operatorname{Aut}^{\alpha+1}(G)$; this minimal $\alpha$ exists by Theorem 2.1 .

The group $G$ is complete if $\tau(G)=0$, i.e., if $\operatorname{Out}(G)=1$.

Many important groups have height one: for example, non-abelian free groups $F$ satisfy Aut $^{2} F=$ Aut $F$; this was shown by J. Dyer and E. Formanek 9 if $F$ has finite rank, and by V. Tolstykh [37] for general $F$. Mapping class groups, simple groups, and arithmetic subgroups of simple Lie groups, are also of height one [6]. The isometry group of a regular, non-rooted tree is complete [39].

In this paper, we are more specifically interested in the automorphism tower of groups acting on rooted, regular trees. These groups are quite interesting in that their automorphism tower often turns out to be quite short $(1,2$ or $\omega$, much less than the upper bound the successor of $2^{\omega}$ given by Theorem [2.11), and can in concrete cases be described explicitly as groups acting on the original tree; that is, automorphisms of these groups are induced by conjugation by tree isometries. Therefore, we deal with the normalizer tower of a group defined as follows.

Definition 1.2. Given a subgroup inclusion $G \leq N$, we define the normalizer tower of $G$ in $N$ as

$$
\begin{gathered}
\operatorname{Norm}_{N}^{0}(G)=G ; \\
\operatorname{Norm}_{N}^{\alpha+1}(G)=\operatorname{Norm}_{N}\left(\operatorname{Norm}_{N}^{\alpha}(G)\right) ;
\end{gathered}
$$

and if $\alpha$ is a limit ordinal, then

$$
\operatorname{Norm}_{N}^{\alpha}(G)=\bigcup_{\beta<\alpha} \operatorname{Norm}_{N}^{\beta}(G) .
$$

To fix notation, let $\mathcal{T}$ be a $d$-regular rooted tree, for some integer $d \geq 2$. Also, let $G$ be a group acting on $\mathcal{T}$. Consider the $d$ subtrees $\mathcal{T}_{1}, \ldots, \mathcal{T}_{d}$ rooted at all vertices neighbouring the root of $\mathcal{T}$. The subtrees $\mathcal{T}_{1}, \ldots, \mathcal{T}_{d}$ are permuted by $G$, and the (set-wise) stabilizer of $\mathcal{T}_{i}$ acts on $\mathcal{T}_{i}$ by restriction. Each of the subtrees $\mathcal{T}_{i}$ is isomorphic to $\mathcal{T}$, and therefore carries an action of $G$ as well as a restricted action of $\operatorname{Stab}_{\mathcal{T}_{i}}(G)$ to the subtree $\mathcal{T}_{i}$. We call $G$ layered if the direct product of $d$ copies of $G$, each acting individually on $\mathcal{T}_{1}, \ldots, \mathcal{T}_{d}$, embeds in $G$, and $G$ permutes transitively the subtrees $\mathcal{T}_{1}, \ldots, \mathcal{T}_{d}$. A first goal of this paper is to give a quite explicit way of calculating the automorphism tower of a layered group (see Section 4):

Theorem 1.3. Let $L$ be a layered group of isometries of the binary tree, set $N=$ $\operatorname{Norm}_{\text {Aut }} \mathcal{T}(L)$, and let $N_{*}$ be the layered closure of $N$, i.e. the smallest layered group containing $N$. Then $\operatorname{Aut}^{i}(L) \leq N_{*}$ for all $i \leq \omega$.

More precisely, let $\mathcal{C}$ be the smallest lattic $\ell^{2}$ of subgroups of Aut $\mathcal{T}$ containing $L, N$ and closed under the operations $G \mapsto G \times G, G \mapsto\{(g, g) \mid g \in G\}, G \mapsto$ $\operatorname{Norm}_{N}(G)$, and $(G, H) \mapsto\left\{g \in H \mid g^{2} \in G\right.$ and $\left.[H, g] \leq G\right\}$ for every $G, H \in \mathcal{C}$ with $G \triangleleft H$. Then $\operatorname{Aut}^{i}(L) \in \mathcal{C}$ for all $i \in \mathbb{N}$.

\footnotetext{
${ }^{2}$ I.e., family of groups closed under the operations $(H, K) \mapsto H \cap K$ and $(H, K) \mapsto\langle H, K\rangle$.
} 
Now consider a slightly weaker condition than being layered: that the intersection of the groups $G$ and $G \times \cdots \times G$ have finite index in both groups. There are some finitely-generated examples of such groups; the best-studied are the "Grigorchuk group" $\mathfrak{G}$ acting on the binary tree [13] and the group $\ddot{\Gamma}$ acting on the ternary tree [16. Both of these groups enjoy many extra properties, such as being torsion, having intermediate word-growth, and being just-infinite. The automorphism group of $\ddot{\Gamma}$ was described in 32 .

A second goal of this paper is to describe quite explicitly all terms in the automorphism tower of these two groups and groups similar to them. The automorphism tower lies inside the full isometry group of the original tree, and the last term of the tower has a natural description:

Theorem 1.4. The group $\ddot{\Gamma}$ defined in [16] has height 2 .

Theorem 1.5. The Grigorchuk group $\mathfrak{G}$ has height $\omega$, and $\operatorname{Aut}^{\omega}(\mathfrak{G})$ is the smallest layered subgroup of Aut $\mathcal{T}$ containing $G$; equivalently, it is the subgroup of Aut $\mathcal{T}$ generated by a copy of $G$ acting below each vertex.

Finally, in a more general direction, we are interested in knowing when the automorphism group of a countable group is again countable, and how far the automorphism tower of such a group grows. An example is the following result, whose proof closely follows [36, Theorem 3.3.1]. We make free use of notions defined in that reference, in particular stationary sets (Definition 3.3.4) and Fodor's lemma (Theorem 3.3.11). Roughly speaking, a stationary set should be thought of as having positive measure, and Fodor's lemma states that a strictly decreasing function on a stationary set has a stationary fiber.

Proposition 1.6. Let $H$ be a countable group. Suppose $G \leq H \leq K$ with $G$ finitely generated and $\operatorname{Cent}_{K}(G)=1$. Then the terms of the normalizer tower of $H$ in $K$ are countable, and therefore the tower has countable height.

Proof. Assume $G=\left\langle s_{1}, \ldots, s_{k}\right\rangle$, and denote the normalizer tower of $H$ by $\left\{N_{\alpha}\right\}$; i.e. $N_{0}=H$ and $N_{\alpha+1}=\operatorname{Norm}_{K}\left(N_{\alpha}\right)$ and $N_{\alpha}=\bigcup_{\gamma<\alpha} N_{\gamma}$ for limit ordinals $\alpha$.

First, we show that $N_{\alpha}$ is countable for all $\alpha<\omega_{1}$, the first uncountable ordinal. This is clearly true for $\alpha=0$. Then, for any $j \in\{1, \ldots, k\}$, we have $s_{j}^{N_{\alpha+1}} \subseteq N_{\alpha}$ and therefore $\left|s_{j}^{N_{\alpha+1}}\right| \leq \aleph_{0}$. This implies $\left[N_{\alpha+1}: \operatorname{Cent}_{N_{\alpha+1}}\left(s_{j}\right)\right] \leq \aleph_{0}$, and hence

$$
\left[N_{\alpha+1}: \bigcap_{i=1}^{k} \operatorname{Cent}_{N_{\alpha+1}}\left(s_{j}\right)\right]=\left[N_{\alpha+1}: \operatorname{Cent}_{N_{\alpha+1}}(G)\right]=\left|N_{\alpha+1}\right| \leq \aleph_{0}
$$

Finally, if $\alpha$ is a countable limit ordinal, then $N_{\alpha}$ is a countable union of countable groups and is therefore countable.

Assume now by contradiction that $N_{\alpha} \neq N_{\beta}$ for all distinct $\alpha, \beta<\omega_{1}$. Let $T$ be the set of countable limit ordinals, which is a stationary subset of $\omega_{1}$. For each $\alpha \in T$ choose an $h_{\alpha} \in N_{\alpha+1} \backslash N_{\alpha}$. Since $\alpha$ is a limit ordinal and $N_{\alpha} \triangleleft N_{\alpha+1}$, we have

$$
G^{h_{\alpha}} \leq N_{\alpha}=\bigcup_{\gamma<\alpha} N_{\gamma}
$$

now for each $j \in\{1, \ldots, k\}$ choose $\gamma_{j}<\alpha$ such that $s_{j}^{h_{\alpha}} \in N_{\gamma_{j}}$, and set $f(\alpha)=$ $\max \left\{\gamma_{j}\right\}$. We have $f(\alpha)<\alpha$ for all $\alpha \in T$, i.e. $f$ is a regressive function $T \rightarrow \omega_{1}$. 
By Fodor's lemma there exists $\gamma<\omega_{1}$ with

$$
S=\{\alpha \in T \mid f(\alpha)=\gamma\} \text { stationary. }
$$

For each $\alpha \in S$ define $\phi_{\alpha}: G \rightarrow H_{\gamma}$ by $x \mapsto x^{h_{\alpha}}$. Any $\phi_{\alpha}$ is determined by the images of $G$ 's generators, so

$$
\left|\left\{\phi_{\alpha} \mid \alpha \in S\right\}\right| \leq\left|H_{\gamma}\right|^{k}<\omega_{1} ;
$$

however $|S|=\omega_{1}$ because $S$ is stationary, so there are $\alpha_{1} \neq \alpha_{2} \in S$ with $\phi_{\alpha_{1}}=\phi_{\alpha_{2}}$; in other words, $1 \neq h_{\alpha_{1}} h_{\alpha_{2}}^{-1} \in \operatorname{Cent}_{K}(W)=1$ and we have reached a contradiction.

Recall that a tree isometry $\alpha \in$ Aut $\mathcal{T}$ is of finite state if its action is induced by a finite transducer, and that the set of finite-state isometries forms a group $R$; see page 336 for the definition.

Corollary 1.7. Let $R$ be the group of finite state isometries. Then $\operatorname{Aut}^{i}(R)$ is countable for all $i \in \mathbb{N}$, so $\tau(R)$ is countable.

Proof. Apply Proposition 1.6 with $K=$ Aut $\mathcal{T}$ and $G$ either the Grigorchuk group (if $d=2$ ) or the Gupta-Sidki group (if $d \geq 3$ ). By Theorem 3.7, we have $\operatorname{Aut}^{\alpha}(R)=$ $\operatorname{Norm}_{K}^{\alpha}(R)$.

As another illustration, consider $G \leq$ Aut $\mathcal{T}$ a finitely generated group acting on the binary tree $\mathcal{T}=\{1,2\}^{*}$, and assume that $G$ contains for all $v \in \mathcal{T}$ an element $g_{v}$ fixing $v 1$ and $v 2$, and having non-conjugate actions on the subtrees rooted at these vertices. Then $\operatorname{Cent}_{\text {Aut } \mathcal{T}}(G)=1$, and Proposition 1.6 applies.

\section{The AUTOMORPhism TOWER}

For a good survey of known results on automorphism groups, see 29]. Even though the rest of the paper does not rely on the considerations in this section, we include some relevant facts here.

First, we note that the automorphism tower has been computed for some classes of groups, and indeed that in many cases the tower has small height. W. Burnside showed in [8, page 95] that $\operatorname{Aut}(G)$ is complete if and only if $G$ is characteristic in Aut $(G)$, i.e. if and only if $G \triangleleft \operatorname{Aut}^{2}(G)$. He then showed (op. cit., next page) that if $G$ is a non-cyclic simple group, then $\tau(G) \leq 1$. Furthermore, the symmetric groups $\operatorname{Sym}(n)$ have height 0 , when $n \neq 6$.

H. Wielandt showed in 38 that the tower of a centerless, finite group is finite. At that time little was known about the height of infinite groups.

A. Rae and J. Roseblade showed in 28 that if $G$ is a Cernikov group, i.e. $G$ is a finite extension of an abelian group and satisfies the minimal condition on subgroups, then $\tau(G)$ is finite. Later J. Dyer and E. Formanek showed in [9] that non-abelian free groups have height 1; i.e. Out $\left(\operatorname{Aut}\left(F_{n}\right)\right)=1$ for $n>1$.

However, groups with infinite height abound among infinite groups; for instance, the infinite dihedral group $D_{\infty}=\left\langle a, b \mid a^{2}, b^{2}\right\rangle$ has an outer automorphism exchanging $a$ and $b$; and Aut $D_{\infty} \cong D_{\infty}$, so $\tau\left(D_{\infty}\right) \geq \omega$. J. Hulse showed in [19] that centerless polycyclic groups have countable height (though not necessarily $\omega$ ). He showed that $D_{\infty}$ has height $\omega+1$, by computing its automorphism tower. It can 
be described using subgroups of $G L\left(2, \mathbb{Z}\left[\frac{1}{2}\right]\right)$ as follows:

$$
\operatorname{Aut}^{\alpha}\left(D_{\infty}\right)= \begin{cases}\left(\begin{array}{cc}
1 & \frac{1}{2^{\alpha}} \mathbb{Z} \\
0 & \pm 1 \\
1 & \mathbb{Z}\left[\frac{1}{2}\right] \\
0 & \pm 1 \\
1 & \mathbb{Z}\left[\frac{1}{2}\right] \\
0 & \pm 2^{\mathbb{Z}}
\end{array}\right) & \text { for } \alpha<\omega \\
& \text { for } \alpha>\omega\end{cases}
$$

The well-definedness of the height of a centerless group is a result of S. Thomas:

Theorem 2.1 (35). Let $G$ be a centerless group. Then there exists an ordinal $\alpha$, less than the successor of $2^{|G|}$, such that $\operatorname{Aut}^{\alpha}(G)=\operatorname{Aut}^{\alpha+1}(G)$.

Furthermore, for any ordinal $\alpha$ there exists a centerless group $G$ with $\tau(G)=\alpha$.

(This last statement was later improved by Just, Shelah and Thomas [20].)

If $G$ has a non-trivial center, more care is needed, since the Aut $^{\alpha}(G)$ no longer form a nested tower. In the definition of $\operatorname{Aut}^{\alpha}(G)$, one replaces the union by a directed limit. J. Hamkins proved in [17] that all groups $G$ have a well-defined height, by actually showing that $\operatorname{Aut}^{\alpha}(G)$ is centerless for some ordinal $\alpha$. Note that for groups possibly with non-trivial center there is no explicit bound on the height of the tower as a function of $|G|$, as in Theorem 2.1.

The considerations we make for the tower of automorphisms also apply to Lie algebras, if "automorphism group" is replaced by "algebra of derivations".

We cannot resist the temptation of repeating the main steps of the proof of Theorem 2.1, since they show a strong relation to groups acting on rooted trees:

Proof of Theorem 2.1 (Sketch). Define inductively the groups $W_{\alpha}$, for ordinals $\alpha$, by $W_{0}=\mathbb{Z} / 2 ; W_{\alpha+1}=W_{\alpha} 2(\mathbb{Z} / 2)$, in which $W_{\alpha}$ embeds as $W_{\alpha} \times 1$; and for limit ordinals $\alpha, W_{\alpha}=\bigcup_{\beta<\alpha} W_{\beta}$.

If $\alpha$ is of the form $1+\beta$, then the normalizer tower of $W_{0}$ in $W_{\alpha}$ stabilizes after exactly $\beta$ steps.

By a result of Fried and Kollár [11, there exists a (usually non-Galois) field $\mathbb{K}$ whose automorphism group is $W_{\beta}$. Then consider the group $G=P G L_{2}(\mathbb{K}) \rtimes W_{0}$. The automorphism tower of $G$ parallels the normalizer tower of $W_{0}$ in $W_{\beta}$, in that Aut $^{\gamma}(G)=P G L_{2}(\mathbb{K}) \rtimes \operatorname{Norm}_{\text {Aut } \mathbb{K}}^{\gamma}\left(W_{0}\right)$ for all $\gamma$. It follows that the automorphism tower of $G$ stabilizes after $\alpha$ steps.

There are still many open questions in the topic of group automorphisms, and in particular, as to how large the automorphism group can be.

Let us mention that there are finitely presented groups with an infinitely generated automorphism group (see for instance [23]), where the group $\left(\mathbb{Z}\left[\frac{1}{2}\right] \times \mathbb{Z}\left[\frac{1}{2}\right]\right) \rtimes \mathbb{Z}^{2}$ is shown to have that property for an appropriate action of $\mathbb{Z}^{2}$; see also [25]. The groups $\mathfrak{G}, \ddot{\Gamma}$ that we consider in this paper also have the property of being finitely generated torsion groups but having an infinitely generated outer automorphism group; however, they are not finitely presented [24, 33].

2.1. Structure of the paper. In Section 3 we recall standard notation for groups acting on rooted trees. We also recall results by Y. Lavreniuk and V. Nekrashevych showing that, under certain conditions often satisfied in practice, the terms of the automorphism tower act on the same tree as the original group. 
In Section 4, we study in some detail the automorphism tower of "layered" groups of isometries of the binary tree, and compute these towers for certain concrete examples.

In Section 5 we study the automorphism tower of the group $\ddot{\Gamma}$ determined in [16], and of its kin the groups $\dot{\Gamma}, \Gamma$ studied in [2]. The last group $\Gamma$ was first studied in 10 . The main result is that, for the groups $\Gamma$ and $\ddot{\Gamma}$, the tower terminates after two steps, i.e. they have height 2.

Finally, in Section 6, we study the automorphism tower of the Grigorchuk group $\mathfrak{G}$ acting on $\mathcal{T}=\{1,2\}^{*}$, and describe the terms of the tower by their action on same tree $\mathcal{T}$. The last term of the tower is shown to be the smallest layered group containing $\mathfrak{G}$.

2.2. Notation. We say that $H$ is characteristic in $G$, written $H \lessdot G$, if $H^{\phi}=H$ for all $\phi \in \operatorname{Aut}(G)$. If $G$ is centerless, we identify $G$ with $\operatorname{lnn}(G)$, and then $H \lessdot G$ if and only if $H \triangleleft \operatorname{Aut}(G)$. We then say that $H$ is absolutely characteristic in $G$ if $H \triangleleft \operatorname{Aut}^{\alpha}(G)$ for all ordinals $\alpha$.

We write $N: Q$ for a split extension of $N$ by $Q$, and $N \cdot Q$ for an non-split extension.

As usual, we write $[a, b]$ for the commutator $a^{-1} b^{-1} a b$, and $a^{b}=b^{-1} a b$ for the conjugation action.

\section{Groups ACting on trees}

Let $X=\{1, \ldots, \mathrm{d}\}$ be an alphabet with $d \geq 2$ elements. The rooted tree $\mathcal{T}$ on $X$ has as its set of vertices the free monoid $X^{*}$, namely the set of finite words $x_{1} \ldots x_{n}$, with $x_{i} \in X$ for all $i$. The tree structure is obtained by connecting $x_{1} \ldots x_{n}$ to $x_{1} \ldots x_{n} x_{n+1}$ for all choices of $x_{i} \in X$; there is a distinguished root vertex $\emptyset$, the empty word. The level of the vertex $v=x_{1} \ldots x_{n}$ is $|v|=n$. All vertices of level $n$ form the $n$th layer $X^{n}$ of $\mathcal{T}$.

The boundary $\partial \mathcal{T}$ of $\mathcal{T}$ is naturally the set $X^{\omega}$ of infinite sequences over $X$. Given a ray $v=x_{1} x_{2} \ldots \in \partial \mathcal{T}$, we denote by $v_{n}$ the truncation $x_{1} \ldots x_{n}$.

For $v \in \mathcal{T}$ of length $n$, we denote by $v \mathcal{T}$ the subtree of $\mathcal{T}$ spanned by all vertices $v w$ with $w \in \mathcal{T}$. Abstractly, it is a tree rooted at $v$, isomorphic to $\mathcal{T}$.

Let $\operatorname{Sym}(X)$ be the symmetric group on $X$. It acts naturally on $\mathcal{T}$ by

$$
\left(x_{1} x_{2} \ldots x_{n}\right)^{\sigma}=x_{1}^{\sigma} x_{2} \ldots x_{n},
$$

and we will always identify $\operatorname{Sym}(X)$ with its image in Aut $\mathcal{T}$.

Let $W$ denote the isomorphism group of $\mathcal{T}$. To avoid confusion with the automorphism group of $W$ itself or of its subgroups, elements of $W$ will be called isometries. Given $g \in G$, there is $\sigma_{g} \in \operatorname{Sym}(X)$ such that $g \sigma_{g}^{-1}$ fixes the vertices of the first level of $\mathcal{T}$; then restriction to subtrees $x \mathcal{T}$ for all $x \in X$ gives states $g @ x \in W$, for all $x \in X$. Conversely, states $g @ x$ and $\sigma_{g} \in \operatorname{Sym}(X)$ can be assembled to give $g \in W$. We therefore have a wreath product structure

$$
W=W \imath_{X} \operatorname{Sym}(X), \quad g \mapsto(g @ 1, \ldots, g @ \mathrm{~d}) \sigma_{g} .
$$

This process can be iterated; for $n \in \mathbb{N}, v \in X^{n}$ and $g \in W$ we write $g @ v$ as the state of $g$ at vertex $v$, and ${ }^{n} \sigma_{g}$ the permutation action of $g$ on $X^{n}$. In other words, $g @ v$ denotes the action $g$ does on the subtree $v \mathcal{T}$ before vertex $v$ is moved by ${ }^{n} \sigma_{g}$.

There is a dual construction: for $v \in \mathcal{T}$ and $g \in W$ we write $v * g$ the isometry of $\mathcal{T}$ that acts as $g$ on $v \mathcal{T}$ and fixes all other vertices. We then have the simple 
Lemma 3.1. @ is a right action of $X^{*}$ on $W$, and $*$ is a left action: for all $g, h \in W$ and $v, w \in X^{*}$ we have

$$
\begin{aligned}
(g @ v) @ w & =g @(v w), & v *(w * g) & =(v w) * g, \\
(g h) @ v & =(g @ v)\left(h @ v^{g}\right), & v *(g h) & =(v * g)(v * h), \\
g & =(v * g) @ v, & g & =\left(\prod_{v \in X^{n}} v *(g @ v)\right){ }^{n} \sigma_{g},
\end{aligned}
$$

where the $v *(g @ v)$ mutually commute when $v$ ranges over the nth layer $X^{n}$.

We also define a variant of the $*$-action by defining isometries $Z g$ for $Z \in$ $\{\dashv, \nabla \triangle\}^{*}$ :

Definition 3.2. For $g \in$ Aut $\mathcal{T}$, we define

$$
\triangle g=(g, \ldots, g), \quad \nabla=\left(1, \ldots, 1, g, g^{-1}\right), \quad \dashv g=(1, \ldots, 1, g)=\mathrm{d} * g,
$$

where a tuple $\left(g_{1}, \ldots, g_{d}\right)$ designates an element $h \in$ Aut $\mathcal{T}$ fixing the first level of $\mathcal{T}$ and satisfying $h @ i=g_{i}$.

For $H \leq$ Aut $\mathcal{T}$ and $Z \in\{\triangle, \nabla,-\}\}$, we define $Z H$ as $\{Z h \mid h \in H\}$. We also let $\times H$ denote the subgroup $H \times \cdots \times H$ generated by $\{\mathrm{i} * h \mid i \in X, h \in H\}$.

We will be concerned with various classes of groups which emerged in recent years as important subgroups of $W$. The vertex stabilizer $\operatorname{Stab}_{G}(v)$ is the subgroup of $G$ fixing $v \in \mathcal{T}$. We make the following definitions: a subgroup $G$ of $W$ is

level-transitive: if $G$ acts transitively on $X^{n}$ for all $n \in \mathbb{N}$;

weakly recurrent: if it is level-transitive, and $\operatorname{Stab}_{G}(x) @ x<G$ for all $x \in$ $X$

recurrent: if it is level-transitive, and $\operatorname{Stab}_{G}(x) @ x=G$ for all $x \in X$;

saturated: if $G$ contains for all $n \in \mathbb{N}$ a characteristic subgroup $H_{n}$ fixing $X^{n}$ such that $H_{n} @ v$ is level-transitive for all $v \in X^{n}$;

weakly branch: if $G$ is level-transitive, and $(v * G) \cap G$ is non-trivial for all $v \in \mathcal{T}$

weakly regular branch: if $G$ is level-transitive, and has a non-trivial normal subgroup $K$ with $x * K<K$ for all $x \in X$;

branch: if $G$ is level-transitive, and $\left\langle(v * G) \cap G: v \in X^{n}\right\rangle$ has finite index in $G$ for all $n \in \mathbb{N}$;

regular branch: if $G$ is level-transitive, and has a finite-index normal subgroup $K$ with $x * K<K$ for all $x \in X$;

pre-layered: if $x * G<G$ for all $x \in X$;

layered: if $G$ is level-transitive and pre-layered.

The subgroup $\operatorname{Rist}_{G}(v)=v * G \cap G$ is called the rigid stabilizer of $v \in \mathcal{T}$. The layered closure of $G$ is the subgroup $G_{*}=\langle v * G: v \in \mathcal{T}\rangle$ of $W$. The closure of $G$ is its topological closure $\widehat{G}$ as a subgroup of $W$; one has

$$
\widehat{G}=\left\{g \in W \mid \text { for all } n \in \mathbb{N} \text { there are } g^{(n)} \in G \text { with } g_{n}^{(n)}=g_{n}\right\} .
$$

The topological closure of a layered group is always $W$.

The pointwise stabilizer in $G$ of the $n$th layer of $\mathcal{T}$ is written $\operatorname{Stab}_{G}(n)$. Clearly $G / \operatorname{Stab}_{G}(n)$ is the permutation group on $X^{n}$ generated by $\left\{g_{n} \mid g \in G\right\}$.

Note that in a weakly branch group all rigid stabilizers are actually infinite. 
Clearly "layered" implies "regular branch", which implies "branch" and "weakly regular branch", each of which imply "weakly branch".

The finitary group $F<W$ is defined as the layered closure of $\operatorname{Sym}(X)$. It is a locally finite, countable subgroup of $W$, and hence is a minimal layered subgroup. Every layered group contains $F$.

Fix an isometry $g \in W$. The activity of $g$ at $v \in \mathcal{T}$ is $\sigma_{g @ v} \in \operatorname{Sym}(X)$. The portrait of $g$ is the activity map $\mathcal{T} \rightarrow \operatorname{Sym}(X), v \mapsto \sigma_{g @ v}$; there is a bijection between $W$ and the set of portraits.

Consider finite-state automata with input alphabet $X$ and states labelled by $\operatorname{Sym}(X)$. The set of portraits defined by such automata defines the group $R$ of finite-state isometries of $\mathcal{T}$. Our main examples of groups, and their automorphism groups, are subgroups of $R$.

For a subgroup $A$ of $\operatorname{Sym}(X)$, consider the subgroup $\widehat{A_{*}}$ of $W$ consisting of those elements whose activity at any vertex is in $A$. If $A$ is transitive on $X$, the resulting group $\widehat{A_{*}}$ is layered. In particular, if $A=\langle(1,2, \ldots, \mathrm{d})\rangle$ and $d$ is prime, then $C=\widehat{A_{*}}$ is a pro- $d$-Sylow of $W$. It consists of all tree isometries whose action below any vertex belongs to $A$.

Lemma 3.3. Let $G$ be any group acting on $\mathcal{T}=X^{*}$. Then the set of pre-layered subgroups of $G$ forms a lattice of groups, closed under taking verbal subgroups.

Proof. If $H, K$ are pre-layered subgroups of $G$, then for any $g \in H \cap K$ we have $x * g \in H \cap K$, and for any $g=h_{1} k_{1} \ldots h_{n} k_{n} \in\langle H, K\rangle$ we have $x * g \in\langle H, K\rangle$ by Lemma 3.1

Finally, for any $W \in F\left(X_{1}, \ldots, X_{n}\right)$ we set $W(H)=\left\{W\left(h_{1}, \ldots, h_{n}\right) \mid h_{i} \in H\right\}$. Given $g=W\left(h_{1}, \ldots, h_{n}\right) \in W(H)$, we have $x * g=W\left(x * h_{1}, \ldots, x * h_{n}\right) \in W(H)$, so $W(H)$ is pre-layered.

Lemma 3.4. If $L$ is a layered group acting on $\mathcal{T}=X^{*}$, then there exists a transitive subgroup $A$ of $\operatorname{Sym}(X)$ such that $L \cong L \imath A$.

Proof. Let $A$ be the quotient of $L$ obtained by restricting the action of $L$ to $X \subset X^{*}$. Since $x * L<L$ for all $x \in X$, and $\operatorname{Stab}_{L}(1) \leq L^{X}$, we have $1 \rightarrow \operatorname{Stab}_{L}(1)=L^{X} \rightarrow$ $L \rightarrow A \rightarrow 1$. Furthermore, every $a \in A$ has a lift $\left(\ell_{1}, \ldots, \ell_{d}\right) a \in L$, and since $\left(\ell_{1}, \ldots, \ell_{d}\right) \in L$ we have $(1, \ldots, 1) a \in L$, defining a splitting of $A$ in $L$.

Proposition 3.5 (R. Möller [26], [Proposition 4). If $L$ is layered and $K \triangleleft L$ is a non-trivial normal subgroup, then $K$ contains $\operatorname{Stab}_{L}(n)^{\prime}$ for some $n \in \mathbb{N}$.

Therefore every quotient of $L$ is abelian by finite.

Assume now that $G$ is generated by a symmetric set $S$. This induces a metric on $G$, defined by

$$
\|g\|=\min \left\{n \mid g=s_{1} \ldots s_{n} \text { with } s_{i} \in S\right\} .
$$

The group $G$ is contracting if there exist constants $\eta<1$ and $K$ such that $\|g @ x\| \leq$ $\eta\|g\|+K$ for all $g \in \operatorname{Stab}_{G}(1)$ and $x \in X \overleftrightarrow{3}$

\footnotetext{
${ }^{3} \mathrm{~A}$ more general notion is usually used [3], namely that there exists $\eta<1, n \in \mathbb{N}$ and $K$ such that $\|g @ u\| \leq \eta^{n}\|g\|+K$ for all $g \in G$ and $u \in X^{n}$. This notion becomes then independent of the choice of the generating set $S$, and there is furthermore an optimal $\eta$, which does not depend on $S$ either.
} 
3.1. Automorphisms and isometries. The following lemma is folklore:

Lemma 3.6. Let $G$ be a weakly branch group. Then the centralizer $\operatorname{Cent}_{W}(G)$ is trivial, and all conjugacy classes of $G$, except $\{1\}$, are infinite.

Proof. Let $a \in W$ be non-trivial; then $a$ moves a point $v \in \mathcal{T}$. Now choose any non-trivial $g \in \operatorname{Rist}_{G}(v)$; then $[a, g] \neq 1$, since it acts similarly to $g$ on $v \mathcal{T}$.

Consider now a non-trivial $g \in G$. It moves a point $v \in \mathcal{T}$, hence its centralizer $\operatorname{Cent}_{G}(g)$ intersects trivially $\operatorname{Rist}_{G}(v)$, which is infinite, so $\operatorname{Cent}_{G}(g)$ has infinite index in $G$ and $g^{G}$ is infinite.

In [21, Y. Lavreniuk and V. Nekrashevych prove

Theorem 3.7. Let $G$ act on $\mathcal{T}$, and suppose $G$ is weakly branch. Then

$$
\operatorname{Norm}_{\text {Homeo }(\partial \mathcal{T})}(G)=\operatorname{Aut}(G) \text {. }
$$

Assume moreover that $G$ is saturated. Then

$$
\operatorname{Norm}_{W}(G)=\operatorname{Aut}(G) \text {. }
$$

Sketch of the proof. The first step is to show that for any $\phi \in \operatorname{Aut}(G)$ and $v \in \mathcal{T}$ there is $w \in \mathcal{T}$ with $\operatorname{Rist}_{G}(v)^{\phi} \geq \operatorname{Rist}_{G}(w)$.

Taking the shortest such $w$ induces a continuous map on the boundary $\partial \mathcal{T}$, which sends $v$ to $w$.

If $G$ is saturated, then $|w|=|v|$, so this map is at each level of the tree a permutation of its vertices. Since $W$ is compact, there is a convergent subsequence of these permutations, which converges to a tree isometry.

The proof is in fact essentially model-theoretic; see 31. Indeed it amounts to showing that the algebraic structure of a saturated weakly branch group is sufficient to reconstruct its action on $\mathcal{T}$.

Lemma 3.8. Let $G$ be a layered group acting on the binary tree. Then $G$ is saturated.

Proof. Define inductively $H_{0}=G$ and $H_{n+1}=\mho_{2}\left(H_{n}\right)$, the subgroup of $H_{n}$ generated by the squares of its elements. Then clearly $H_{n}$ is characteristic in $G$ for all $n$, and since $G$ is layered, $H_{n}$ contains for all $g \in G$ the element

$$
\left(\left(\left(\ldots(g, 1)^{\sigma}, 1\right)^{\sigma}, \ldots, 1\right)^{\sigma}\right)^{2^{n}}=(g, \ldots, g)
$$

with $2^{n}$ copies of $g$; therefore $H_{n}$ fixes $X^{n}$ and acts transitively on every subtree at level $n$.

Lemma 3.9. Let $G$ be a group acting on the binary tree. If $G$ contains a weakly branch group, then $G$ is weakly branch.

If $G$ contains a subgroup $H$ such that $\left(\mho_{2}\right)^{n}(H)$ acts transitively on every subtree at level $n$, for all $n \in \mathbb{N}$, then $G$ is saturated.

Proof. The first statement follows directly from the definition: if $G$ contains the weakly branch subgroup $H$, then $(v * H) \cap H \neq 1$ for all $v \in \mathcal{T}$, and hence $(v * G) \cap G \neq$ 1 for all $v \in \mathcal{T}$.

For the second statement, $\left(\mho_{2}\right)^{n}(G)$ fixes $X^{n}$, and also acts transitively on every subtree at level $n$.

Proposition 3.10. The groups $F$ and $W$ are layered and saturated. 
Proof. Branchness is clear, since we have $\operatorname{Rist}_{W}(v)=v * W$ and $\operatorname{Rist}_{F}(v)=v * F$.

To prove saturatedness, we invoke Theorem 3.11 indeed choose $x \in X$ and write $W=\operatorname{Rist}_{W}(x) 2_{X} \operatorname{Sym}(X)$; then $H_{1} \lessdot W$ may be chosen to be its base group $W^{X}$. Inductively, let $K$ be the copy of $H_{n-1}$ inside $\operatorname{Rist}_{W}(x)=x * W$; then $H_{n} \lessdot W$ may be chosen as $K^{X}$, characteristic in $W^{X}$ which is itself characteristic in $W$.

The same argument applies to $F$.

Wreath products exhibit a form of rigidity which was already noted independently by a number of authors:

Theorem 3.11 (P. Neumann, 27; Y. Bodnarchuk, 5]; P. Lentoudis and J. Tits, 22]). If $A, B$ are non-trivial groups with $B$ acting on a set $X$, then the base group $A^{X}$ is characteristic in the wreath product $A 2_{X} B$, unless $A=\mathbb{Z} / 2$ and $B$ has an abelian subgroup $B_{0}$ of index 2 containing unique square roots of its elements.

Furthermore, in some specific examples, a strengthening of this rigidity has been obtained: in essence, not only are all group isomorphisms induced by a tree isomorphism, but moreover there is a unique minimal tree carrying the group's action. R. Grigorchuk and J. S. Wilson showed that if $G$ is a branch group acting on a group $\mathcal{T}$ satisfying two technical conditions, and if $G$ also acts on another tree $\mathcal{T}^{\prime}$ as a branch group, then the actions on $\mathcal{T}$ and $\mathcal{T}^{\prime}$ are intertwined by map $\mathcal{T}^{\prime} \rightarrow \mathcal{T}$ defined by the erasing of some levels in $\mathcal{T}^{\prime}$.

In contrast, finite groups behave in a manner very different from the groups we are interested in:

Theorem 3.12. If the finite group $G$ acts on $\mathcal{T}$, then $\operatorname{Cent}_{W}(G)$ is uncountable.

Proof. For simplicity we prove the result only for $\mathcal{T}$ the binary tree $\{1,2\}^{*}$. We proceed by induction, first on $|G|$, and then on the smallest level at which a nontrivial activity occurs, i.e. the first $k$ such that $G$ is not a subset of $\operatorname{Stab}_{W}(k)$.

If $|G|=1$, then $\operatorname{Cent}_{W}(G)=W$ and we are done. If $G \leq \operatorname{Stab}_{W}(1)$, then consider the projections $H_{1}, H_{2}$ of $G$ defined by restriction to the respective subtrees rooted at level 1 . These are finite groups, of size at most $|G|$, and at least one of $H_{1}, H_{2}$, say $H_{1}$, has already been covered by induction; therefore $\operatorname{Cent}_{W}(G)$ contains Cent $_{W}\left(H_{1}\right) \times\{1\}$ and is uncountable.

We may therefore assume that $G$ contains an element $g=\left(g_{1}, g_{2}\right) \sigma$; also let $H$ be the projection of $\operatorname{Stab}_{G}(1)$ on the subtree rooted at 1 . Set $Z=\operatorname{Cent}_{W}(H)$; by induction, $Z$ is uncountable. Furthermore, $\operatorname{Stab}_{G}(1) \leq H \times H^{g_{2}^{-1}}$, so the centralizer of $\operatorname{Stab}_{G}(1)$ contains $Z \times Z^{g_{2}^{-1}}$.

The centralizer of $g$ contains all elements $\left(z, z^{g_{2}^{-1}}\right)$ with $z \in \operatorname{Cent}_{W}\left(g_{1} g_{2}\right)$; and since $g^{2}=\left(g_{1} g_{2}, g_{2} g_{1}\right) \in \operatorname{Stab}_{G}(1)$, we see that $\operatorname{Cent}_{W}(G)$ contains all $\left(z, z^{g_{2}^{-1}}\right)$ for $z \in Z$; therefore $\operatorname{Cent}_{W}(G)$ is uncountable.

Also consider the following example of a group with uncountable normalizer: let $U$ be an infinite minimal connecting set of vertices of the tree $\mathcal{T}$, i.e. a subset of $\mathcal{T}$ 's vertices that intersects any infinite ray. For every $u \in U$, let $H(u)$ be a subgroup of Aut $(\mathcal{T})$. Let $G$ be the group generated by all $u * H(u)$, with $u$ ranging over $U$. Then $G$ is isomorphic to the direct sum of the $H(u)$ 's, and the normalizer of $G$ in $W$ contains the cartesian product of the $H(u)$ 's.

Let us finally note a result related to Theorem 3.7 and expressed in terms of commensurators: an almost automorphism of $G$ is an automorphism between two 
finite-index subgroups of $G$. Two almost automorphisms are equivalent if they agree on a finite-index subgroup of $G$. The set of equivalence classes of almost automorphisms carries a natural group structure, and is called the abstract commensurator of $G$.

On the other hand, if $G$ is a subgroup of $K$, then the commensurator of $G$ in $K$ is $\left\{k \in K \mid\left[G: G \cap G^{k}\right]<\infty,\left[G^{k}: G \cap G^{k}\right]<\infty\right\}$.

Theorem 3.13 (C. Röver, 30]). The abstract commensurator of a weakly branch group $G$ acting on $T$ is isomorphic to the commensurator of $G$ in $\mathrm{Homeo}(\partial \mathcal{T})$.

3.2. The automorphism tower. Our first purpose is, given a suitable $G$, to identify a group $G_{*}$ that is much smaller than $W$ (i.e., for instance, is countable), but still is large enough to contain the whole automorphism tower of $G$. This could be interpreted as a strengthening of Theorem 3.7

For any $n \in \mathbb{N}$ set $G_{n}=\langle v * G: v \in \mathcal{T},|v| \leq n\rangle$, and $G_{*}=\bigcup_{n \geq 0} G_{n}$. The following is immediate:

Lemma 3.14. Assume $G$ is weakly branch and recurrent. Then $G_{n}$ is weakly branch for all $n$, and the $G_{n}$ form an ascending tower. Its limit $G_{*}$ is layered, and is the smallest layered group containing $G$. If $G$ is countable, then so is $G_{*}$.

Theorem 3.15. If $G$ is a finitely generated group with a recurrent, saturated, and weakly branch action on $\mathcal{T}$, and if $G$ satisfies $G_{*}=\bigcup_{n \in \mathbb{N}} \operatorname{Norm}^{n}(G)$, then $G_{*}$ is complete; in other words, $G$ has height at most $\omega$.

We remark that the condition $G_{*}=\bigcup_{n \in \mathbb{N}} \operatorname{Norm}^{n}(G)$ implies that for all $n \in \mathbb{N}$ there are $k, \ell \in \mathbb{N}$ such that $\times^{k} G<\operatorname{Norm}^{n}(G)<\left(\times{ }^{\ell} G\right)\left({ }^{\ell} C_{2}\right)$, since $G$ is finitely generated.

Proof. Take $\phi \in \operatorname{Aut}\left(G_{*}\right)$. Then by Theorem 3.7 we have $\phi \in$ Aut $\mathcal{T}$, and similarly $\psi=\phi^{-1} \in$ Aut $\mathcal{T}$. Since by definition $G$ is finitely generated, there exists $n \in \mathbb{N}$ such that $\phi(G) \leq \operatorname{Norm}^{n}(G)$ and $\psi(G) \leq \operatorname{Norm}^{n}(G)$.

Since $G_{*}=\bigcup_{m \in \mathbb{N}} G_{m}$, there is also an $m \in \mathbb{N}$ such that $\phi(G) \leq G_{m}$ and $\psi(G) \leq G_{m}$.

Let $u, \ldots, v$ be all the vertices on level $m$ of $\mathcal{T}$, and let us decompose $\phi=$ $\left(\phi_{u}, \ldots, \phi_{v}\right) \sigma$ and $\psi=\left(\psi_{u}, \ldots, \psi_{v}\right) \sigma^{-1}$. Since $G$ is recurrent, for any $g \in G$ there exists a $g^{\prime} \in G$ with level- $m$ decomposition $(g, *, \ldots, *)$. Then $\left(g^{\prime}\right)^{\phi}=$ $\left(g^{\phi_{u}}, *, \ldots, *\right)^{\sigma}$ belongs to $G$, and therefore $\phi_{u}$ induces by conjugation an endomorphism of $G$.

Then $(g, *, \ldots, *)=g^{\prime}=\phi \psi\left(g^{\prime}\right)=\left(\phi_{u}(*), *, \ldots, *\right)$, and therefore $\phi_{u}(G)=G$, so $\phi_{u}$ induces an automorphism of $G$. We have shown $\operatorname{Aut}\left(G_{*}\right) \leq(\operatorname{Aut}(G) F)_{*}=$ $G_{*}$.

\section{LAYERED GROUPS}

We describe in this section the automorphism tower of a layered group $L$ acting on the binary tree $\mathcal{T}=X^{*}$, with $X=\{1,2\}$; recall that such a group has a decomposition $L=(L \times L) \rtimes\langle\sigma\rangle$, where $\sigma$ denotes the transposition of the top two branches of the binary tree.

Denote $\operatorname{Aut}(L)$ by $A$. By Lemma 3.8 and Theorem 3.7 every automorphism $\alpha$ of $L$ acts on the binary tree, and therefore we have $A \leq(W \times W) \rtimes\langle\sigma\rangle$. 
Then, given $\alpha=\left(\alpha_{1}, \alpha_{2}\right) \sigma^{i} \in A$, we have $(\ell, 1)^{\alpha}=\left(\ell^{\alpha_{1}}, 1\right)^{\sigma^{i}} \in L$, and similarly $(1, \ell)^{\alpha} \in L$ so $\alpha_{i} \in A$ for all $i \in X$ and $A \leq(A \times A) \rtimes\langle\sigma\rangle$. Furthermore, $\sigma^{\alpha}=\sigma^{1-i}\left(\alpha_{2}^{-1} \alpha 1, \alpha_{1}^{-1} \alpha_{2}\right) \sigma^{i} \in L$, so $\alpha_{2}^{-1} \alpha_{1} \in L$ and $A \leq(A \times 1) L$. In other words, $\alpha$ can be written as a (possibly infinite) product

$$
\alpha=\cdots\left(1^{n} * \ell_{n}\right) \cdots\left(1 * \ell_{1}\right) \ell_{0},
$$

for some $\ell_{n} \in L$. On the other hand, such a product defines an endomorphism (but not necessarily an automorphism) of $L$, by conjugation.

Note also that $\alpha$ is a finite state provided the sequence $\left(\ell_{n}\right)$ is ultimately periodic (see [7]). That same paper shows that, for $F$ the finitary group, Aut $(F)$ contains a copy of $W$ and is therefore uncountable.

We mention in passing another proof that $\operatorname{Aut}(F)$ is uncountable: recall that an automorphism $\phi$ of a group $G$ is locally inner if $\phi_{\mid X}$ is inner for every finite $X \subset G$. Let $\operatorname{Linn}(G)$ denote the group of locally inner automorphisms of $G$.

Proposition 4.1 ([18, page 37). Let $G$ be a countably infinite, locally finite group, and suppose that $\operatorname{Cent}_{G}(F)$ is not central in $G$, for all finite subgroups $F$ of $G$. Then $|\operatorname{Linn}(G)|=2^{\aleph_{0}}$.

It is, however, unknown whether $|\operatorname{Aut}(G)|=2^{\aleph_{0}}$ for all countably infinite, locally finite groups $G$.

Definition 4.2. For a layered group $L$, we write $\operatorname{Aut}^{i}(L)$ for the $i$-th term in the automorphism tower of $L$. For groups $K \triangleleft G$, we write

$$
\Omega(G, K)=\left\{v \in G \mid v^{2} \in K \text { and }[G, v] \leq K\right\} .
$$

We shall be concerned, in this section, with groups $G \leq W$ that admit a decomposition

$$
G=(\times U)\langle\sigma\rangle(\triangle S),
$$

where $U \triangleleft S$ is maximal, and $G$ contains a fixed layered group $L$. By Theorem 3.7 we have Aut $G=\operatorname{Norm}_{W}(G)=$ : Norm $G$. The form (3) is preserved by some important operations, namely if $G=(\times U)\langle\sigma\rangle(\triangle S)$ and $H=(\times V)\langle\sigma\rangle(\triangle T)$, then

(Lemma 4.6)

$$
G \cap H=(\times(U \cap V))\langle\sigma\rangle(\triangle(S \cap T)),
$$

(Theorem 4.4)

$$
\begin{aligned}
\Omega(G, H) & =(\times(U \cap \Omega(S, V)))\langle\sigma\rangle(\triangle(\Omega(S, T))), \\
\operatorname{Norm}(G) & =(\times \Omega(S, U))\langle\sigma\rangle(\triangle(\operatorname{Norm} U \cap \operatorname{Norm} S)) .
\end{aligned}
$$

Lemma 4.3. Consider a group $H=(\times K)\langle\sigma\rangle$. Then

$$
\operatorname{Norm}(H)=(\times K)\langle\sigma\rangle(\triangle \operatorname{Norm}(K))
$$

where $\times K$ is the largest geometrically decomposable subgroup of $\operatorname{Norm}(H)$ and $\triangle \operatorname{Norm}(K)$ is the largest diagonal subgroup of $\operatorname{Norm}(H)$.

Proof. Choose $\alpha=\left(\alpha_{1}, \alpha_{2}\right) \sigma^{i} \in \operatorname{Norm}(H)$. Then $\left(\alpha_{1}, \alpha_{2}\right) \in \operatorname{Norm}(H)$, and $\alpha_{1}, \alpha_{2} \in$ $\operatorname{Norm}(K)$. Thus, $\alpha \in \times \operatorname{Norm}(K)\langle\sigma\rangle$ and $\left[\left(\alpha_{1}, \alpha_{2}\right), \sigma\right]=\left(\alpha_{1}^{-1} \alpha_{2}, \alpha_{2}^{-1} \alpha_{1}\right) \in \times K$. Therefore, $\alpha_{2}=\alpha_{1} k$ for some $k \in K$; that is, $\alpha=\left(\alpha_{1}, \alpha_{1} k\right) \sigma^{i}$. Since $\triangle \alpha_{1} \in$ $\operatorname{Norm}(H)$ for all $\alpha_{1} \in \operatorname{Norm}(K)$, the decomposition

$$
\operatorname{Norm}(H)=(\times K)\langle\sigma\rangle(\triangle \operatorname{Norm}(K))
$$

follows.

The rest of the assertions are easy. 
Theorem 4.4. Consider $R=(\times U)\langle\sigma\rangle(\triangle S)$, where $U$ is a normal subgroup of $S$. Then

$$
\operatorname{Norm}(R)=(\times V)\langle\sigma\rangle(\triangle T)
$$

where $V=\Omega(S, U)$ and $T=\operatorname{Norm}(U) \cap \operatorname{Norm}(S)$.

Furthermore, $V$ is a normal subgroup of $T,[\times V, R] \leq(\times U)(\triangle V)$, the quotient group $V / U$ is a $T / S$-module, and

$$
\operatorname{Norm}(R) / R \cong(V / U)(T / S) .
$$

Proof. First, it is clear that $V$ is a normal subgroup of $T$ and that $\times V$ normalizes both $\times U$ and $\triangle S$.

Choose $(v, 1) \in \times V$; then $[(v, 1), \sigma]=\left(v^{-1}, v\right)=\left(v^{-2}, 1\right)(v, v)$, where by the definition of $V$ we have $v^{-2} \in U$ and $(v, v) \in \triangle S$. Therefore $\times V \leq \operatorname{Norm}(R)$. Easily, $\triangle T \leq \operatorname{Norm}(R)$.

Next choose $\beta \in \operatorname{Norm}(R)$. Then we may assume $\beta=\left(\beta_{1}, \beta_{2}\right)$. As $\times U$ is normal in $R$, we conclude that $\beta_{1}, \beta_{2} \in \operatorname{Norm}(U)$ and commutation with $\triangle S$ shows that $\beta_{1}, \beta_{2} \in \operatorname{Norm}(U S)=\operatorname{Norm}(S)$. Thus, $\beta_{1}, \beta_{2} \in T$ and

$$
\operatorname{Norm}(R) \leq(\times T)\langle\sigma\rangle \text {. }
$$

Now, $\sigma^{\beta}=\left(\beta_{1}^{-1} \beta_{2}, \beta_{2}^{-1} \beta_{1}\right) \sigma=\left(u_{1} s, u_{2} s\right) \sigma$ for some $u_{1}, u_{2} \in U, s \in S$. Since $\sigma^{2}=1$, we have $u_{2} s=\left(u_{1} s\right)^{-1}=u_{1}^{-s} s^{-1}$, and therefore

$$
s^{2}=u_{2}^{-1} u_{1}^{-s} \in U, \quad \beta_{2}=\beta_{1} u_{1} s .
$$

Thus, $\beta=\left(\triangle \beta_{1}\right)\left(1, u_{1} s\right)$ with $\beta_{1} \in T$. Since we have already shown $\triangle T \leq$ $\operatorname{Norm}(R)$, and as $1 \times U \leq R$, we may assume $\beta=\left(1, u_{1} s\right)$, and as $1 \times U \leq R$ we may assume further that $\beta=(1, s)$. Now, $[\triangle S, \beta]=1 \times[S, s] \leq R$ and therefore $[S, s] \leq U$. Hence, $s \in \Omega(S, U)=V$ and $\operatorname{Norm}(R)=(\times V)\langle\sigma\rangle(\triangle T)$.

We then have

$$
\begin{aligned}
{[\times V, R] } & =[\times V, \times U][\times V, \triangle S\langle\sigma\rangle] \\
& =(\times[V, U])(\times[V, S])\left\{\left(v^{-1}, v\right) \mid v \in V\right\} \\
& \leq(\times U)(\triangle V),
\end{aligned}
$$

since $[V, S],\left(V^{2}, 1\right) \leq U$. We observe the following facts:

$$
\begin{gathered}
(\triangle T) \cap R=(\triangle T) \cap(\triangle(U S))=\triangle(T \cap S)=\triangle S, \\
((\times V)\langle\sigma\rangle) \cap R=(\times U)(\triangle V)\langle\sigma\rangle,
\end{gathered}
$$

$V / U$ is a $T$-module and the kernel of the action contains $S$.

Finally, let $W$ be a transversal of $U$ in $V$ and let $X$ be a transversal of $S$ in $T$. Then $(W \times 1)(\triangle X)$ is a transversal of $R$ in $\operatorname{Norm}(R)$, and hence $\operatorname{Norm}(R) / R \cong$ $(V / U)(T / S)$.

Remark 4.5. Suppose $\operatorname{Norm}(S)=S$. Then

$$
\operatorname{Norm}^{i}(R)=\left(\times U_{i}\right)\langle\sigma\rangle(\triangle S),
$$

where $U_{0}=U$ and $U_{i}=\Omega\left(S, U_{i-1}\right)$. The subgroups $U_{i}$ form a hypercentral series of $S$ with respect to $U$.

In particular, if $S$ is finitely generated, then $\operatorname{Norm}^{\omega}(R)=\operatorname{Norm}^{\omega+1}(R)$, because letting $U_{\omega}=\bigcup_{n \in \mathbb{N}} U_{n}$ we have $\Omega\left(S, U_{\omega}\right)=U_{\omega}$. 


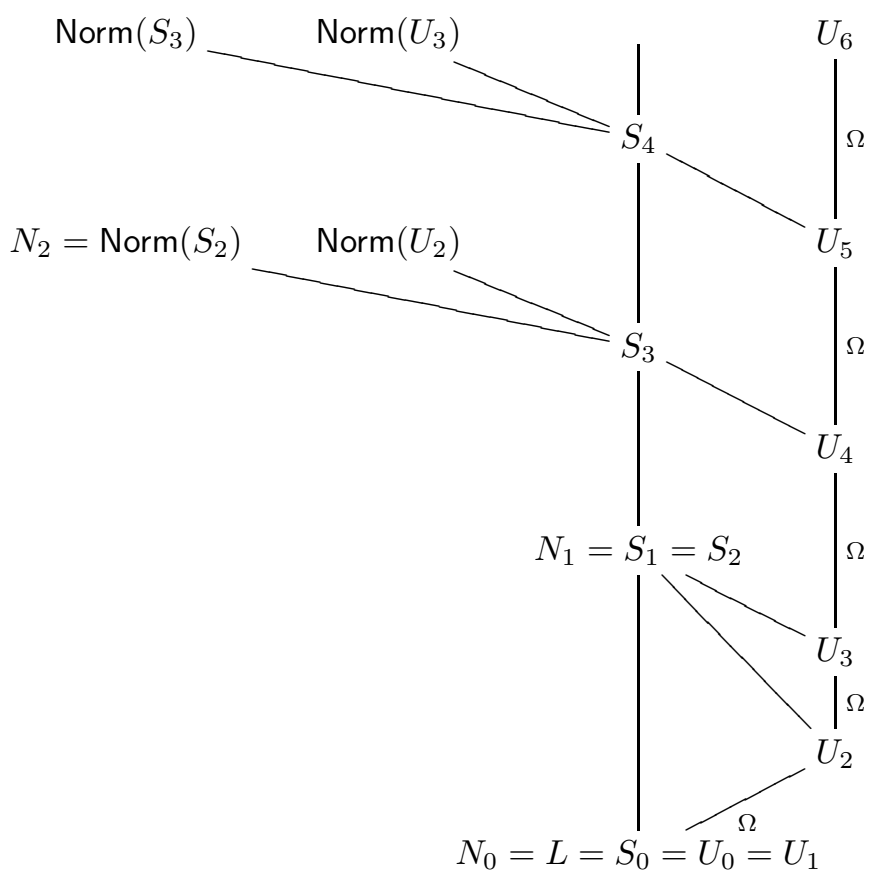

FiguRE 1. Some groups appearing in Theorem 4.7

Lemma 4.6. Consider $G=(\times U)\langle\sigma\rangle(\triangle S)$, and let $H=(\times V)\langle\sigma\rangle(\triangle T)$ be a normal subgroup of $G$. Then

$$
\Omega(G, H)=(\times(U \cap \Omega(S, V)))\langle\sigma\rangle(\triangle \Omega(S, T)) .
$$

Proof. By assumption we have $V \triangleleft S, T \triangleleft S, U \triangleleft S, V \triangleleft T$, and $U \leq \Omega(T, V)$.

Set $Q=\Omega(G, H)$. First, consider $y=(x, 1)$ with $x \in U \cap \Omega(S, V)$. Then $x^{2} \in V$ and $[x, S] \subseteq V$ so $y^{2} \in H$ and $[y, G] \in H$, so $y \in Q$. Conversely, if $y=(1, x) \in Q$, then $[y, G] \in H,[y, \sigma] \in H$ and $y^{2} \in H$ imply $x \in U \cap \Omega(S, V)$.

Next consider $y=(x, x)$ with $x \in \Omega(S, T)$. Then $x^{2} \in T$ and $[x, S] \subseteq T$ so $y^{2} \in H$ and $[y, G] \in H$, so $y \in Q$. Conversely, if $y=(x, x) \in Q$, then $[y, G] \in H$ and $y^{2} \in H$ imply $x \in \Omega(S, T)$.

Theorem 4.7. Let $L$ be a layered group, $L=(\times L)\langle\sigma\rangle$ and let $\left(N_{i}=\operatorname{Norm}^{i}(L)\right)_{i \geq 0}$ be the normalizer tower of $L$. Then we have the equations (see Figure 1)

$$
\begin{aligned}
N_{0} & =U_{0}=S_{0}=L, \\
N_{i} & =\left(\times U_{i}\right)\langle\sigma\rangle\left(\triangle S_{i}\right), \\
U_{i} & =\Omega\left(S_{i-1}, U_{i-1}\right), \\
S_{i} & =\operatorname{Norm}\left(U_{i-1}\right) \cap \operatorname{Norm}\left(S_{i-1}\right) .
\end{aligned}
$$

For $1 \leq i \leq 4$, the description of $N_{i}$ by the pair $\left(U_{i}, S_{i}\right)$ is as follows:

$$
\begin{array}{ll}
U_{1}=(\times L)\langle\sigma\rangle=L, & S_{1}=N_{1} ; \\
U_{2}=(\times L)\langle\sigma\rangle\left(\triangle U_{2}\right), & S_{2}=N_{1} ;
\end{array}
$$




$$
\begin{array}{ll}
U_{3}=(\times L)\langle\sigma\rangle\left(\triangle U_{3}\right), & S_{3}=\left(\times U_{2}\right)\langle\sigma\rangle\left(\triangle N_{1}\right)=N_{2} ; \\
U_{4}=\left(\times U_{2}\right)\langle\sigma\rangle\left(\triangle \Omega\left(N_{1}, U_{3}\right)\right), & S_{4}=\left(\times \Omega\left(U_{3}, U_{1}\right)\right)\langle\sigma\rangle\left(\triangle N_{1}\right) .
\end{array}
$$

Furthermore, the outer-normalizer groups are

$$
\begin{aligned}
& N_{2} / N_{1} \cong U_{2} / U_{1}, \\
& N_{3} / N_{2} \cong\left(U_{3} / U_{2}\right)\left(N_{2} / N_{1}\right), \\
& N_{4} / N_{3} \cong\left(U_{4} / U_{3}\right)\left(\Omega\left(U_{3}, L\right) / U_{2}\right)\left(N_{2} / N_{1}\right),
\end{aligned}
$$

where $U_{i+1} / U_{i}$ and $\Omega\left(U_{3}, L\right) / U_{2}$ are elementary abelian 2-groups.

Proof. Since $L=(\times L)\langle\sigma\rangle(\triangle L)$, the general form of the normalizer subgroups $\operatorname{Norm}^{i}(L)=\left(\times U_{i}\right)\langle\sigma\rangle\left(\triangle S_{i}\right)$ is determined by Theorem 4.4. We compute for $i \leq 4$ :

$\mathbf{i}=1$. We have $N_{1}=\left(\times U_{1}\right)\langle\sigma\rangle\left(\triangle S_{1}\right)$, with $U_{1}=\Omega(L, L)=L=U_{0}$ and $S_{1}=$ $\operatorname{Norm}(L) \cap \operatorname{Norm}(L)=\operatorname{Norm}(L)=N_{1}$. We do not obtain any information about the outer-normalizer group $N_{1} / N_{0}$, since

$$
N_{1} / N_{0} \cong\left(U_{1} / U_{0}\right)\left(S_{1} / S_{0}\right)=S_{1} / S_{0} \cong N_{1} / N_{0} .
$$

$\mathbf{i}=2$. First, $N_{2}=\left(\times U_{2}\right)\langle\sigma\rangle\left(\triangle S_{2}\right)$, with $U_{2}=\Omega\left(N_{1}, U_{1}\right)$ and $S_{2}=\operatorname{Norm}\left(U_{1}\right) \cap$ $\operatorname{Norm}\left(S_{1}\right)$.

Second, $S_{2}=\operatorname{Norm}(L) \cap \operatorname{Norm}\left(N_{1}\right)=N_{1} \cap N_{2}=N_{1}$.

Third, $N_{0}=L=L(\triangle L) \leq U_{2}=\Omega\left(N_{1}, U_{1}\right) \leq N_{1}=L\left(\triangle N_{1}\right)$. By Lemma 4.6. $U_{2}=L(\triangle W)$, with $W=\Omega\left(N_{1}, N_{0}\right)=\Omega\left(N_{1}, U_{1}\right)=U_{2}$; therefore,

$$
U_{2}=L\left(\triangle U_{2}\right)=U_{1}\left(\triangle U_{2}\right) .
$$

Fourth, $\operatorname{Norm}\left(U_{2}\right)=(\times V)\langle\sigma\rangle(\triangle Y)$, with $V=\Omega\left(U_{2}, U_{1}\right)=U_{2}$ and $Y=$ $\operatorname{Norm}\left(U_{1}\right) \cap \operatorname{Norm}\left(U_{2}\right)=N_{1} \cap \operatorname{Norm}\left(U_{2}\right)=N_{1}$, and therefore $N_{2}=\operatorname{Norm}\left(U_{2}\right)$.

Finally, $N_{2} / N_{1} \cong\left(U_{2} / U_{1}\right)\left(S_{2} / S_{1}\right)=U_{2} / U_{1}$, an elementary abelian 2-group.

$\mathbf{i}=3$. First, $N_{3}=\left(\times U_{3}\right)\langle\sigma\rangle\left(\triangle S_{3}\right)$, with $U_{3}=\Omega\left(S_{2}, U_{2}\right)$ and $S_{3}=\operatorname{Norm}\left(U_{2}\right) \cap$ $\operatorname{Norm}\left(S_{2}\right)$.

Second, $S_{3}=N_{2} \cap \operatorname{Norm}\left(S_{2}\right)=N_{2}$.

Third, $U_{3}=\Omega\left(N_{1}, U_{2}\right)$ and $L\left(\triangle U_{2}\right) \leq U_{3} \leq N_{1}=L\left(\triangle N_{1}\right)$, so $U_{3}=L(\triangle W)$ where $W=\Omega\left(N_{1}, U_{2}\right)=U_{3}$; and hence, $U_{3}=L\left(\triangle U_{3}\right)$.

Finally, $N_{3} / N_{2} \cong\left(U_{3} / U_{2}\right)\left(S_{3} / S_{2}\right)=\left(U_{3} / U_{2}\right)\left(N_{2} / N_{1}\right)$ where, as we had shown, $N_{2} / N_{1} \cong U_{2} / U_{1}$.

$\mathbf{i}=4$. First, $N_{4}=\left(\times U_{4}\right)\langle\sigma\rangle\left(\triangle S_{4}\right)$, with $U_{4}=\Omega\left(S_{3}, U_{3}\right)=\Omega\left(N_{2}, U_{3}\right)$ and $S_{4}=$ $\operatorname{Norm}\left(U_{3}\right) \cap \operatorname{Norm}\left(S_{3}\right)$.

Second, $S_{4}=\operatorname{Norm}\left(U_{3}\right) \cap \operatorname{Norm}\left(N_{2}\right)=\operatorname{Norm}\left(U_{3}\right) \cap N_{3}$.

Third, from $U_{3}=(\times L)\langle\sigma\rangle\left(\triangle U_{3}\right)$ we find

$$
\operatorname{Norm}\left(U_{3}\right)=(\times W)\langle\sigma\rangle\left(\triangle\left(\operatorname{Norm}(L) \cap \operatorname{Norm}\left(U_{3}\right)\right)\right),
$$

with $W=\Omega\left(U_{3}, L\right)$. Then $\operatorname{Norm}(L) \cap \operatorname{Norm}\left(U_{3}\right)=N_{2} \cap \operatorname{Norm}\left(U_{3}\right)=S_{3} \cap \operatorname{Norm}\left(U_{3}\right)=$ $N_{2}$, and $\operatorname{Norm}\left(U_{3}\right)=(\times W)\langle\sigma\rangle\left(\triangle N_{2}\right)$. Since $W=\Omega\left(U_{3}, L\right) \leq U_{3}=\Omega\left(N_{1}, L\right)$, we conclude $\operatorname{Norm}\left(U_{3}\right) \leq N_{3}$; and hence, $S_{4}=\operatorname{Norm}\left(U_{3}\right)$.

Fourth, $U_{4}=\Omega\left(N_{2}, U_{3}\right)$. Since

$$
U_{3}=(\times L)\langle\sigma\rangle\left(\triangle U_{3}\right) \leq U_{4} \leq N_{2}=\left(\times U_{2}\right)\langle\sigma\rangle\left(\triangle N_{1}\right)
$$

and $\left(\times U_{2}\right) \leq \Omega\left(N_{2}, U_{2}\right) \leq \Omega\left(N_{2}, U_{3}\right)$, we have $\left(\times U_{2}\right)\langle\sigma\rangle\left(\triangle U_{3}\right) \leq U_{4} \leq N_{2}=$ $\left(\times U_{2}\right)\langle\sigma\rangle\left(\triangle N_{1}\right)$. As in the previous case, $U_{4}=\left(\times U_{2}\right)\langle\sigma\rangle(\triangle V)$, with $V=\Omega\left(N_{1}, U_{3}\right)$. 
Finally, $N_{4} / N_{3} \cong\left(U_{4} / U_{3}\right)\left(S_{4} / S_{3}\right)$, and

$$
\begin{aligned}
S_{4} / S_{3} & =\operatorname{Norm}\left(U_{3}\right) / N_{2} \cong\left((\times W)\langle\sigma\rangle\left(\triangle N_{2}\right)\right) /\left(\left(\times U_{2}\right)\langle\sigma\rangle\left(\triangle N_{1}\right)\right) \\
& \cong\left(W / U_{2}\right)\left(N_{2} / N_{1}\right) .
\end{aligned}
$$

It was shown in 34 that, up to conjugation, the maximal 2-subgroups $M$ of $\operatorname{Aut}(\mathcal{T})$ can be of two types. First, subgroups of the form $M=M_{1} \times M_{2}$, where $M_{1}, M_{2}$ are two non-conjugate maximal 2-subgroups of Aut $(\mathcal{T})$. The second type consists of layered groups $M=(M \times M)\langle\sigma\rangle$.

Corollary 4.8. Suppose $L$ is a maximal 2-subgroup of $\operatorname{Aut}(\mathcal{T})$ which is layered. Then $N_{2}(L)=N_{1}(L)$, i.e. $L$ has height at most 2 .

We now proceed towards the proof of Theorem 1.3 .

Lemma 4.9. Let $L$ be a layered group. Then for every $i \in \mathbb{N}$ we have $\operatorname{Rist}_{\text {Aut }^{i}(L)}(w)$ $=L$ for all $w \in \mathcal{T}$ with $|w| \geq i$.

The subgroup $\times^{i} L$ is characteristic in $\operatorname{Aut}^{i}(L)$.

Proof. For $i=0$ the assertions are clear, while for $i=1$ they follow from Lemma4.3. We then prove both claims simultaneously by induction.

Assume that $\operatorname{Rist}_{\operatorname{Aut}^{i}(L)}(w)=L$ for all $w \in \mathcal{T}$ with $|w| \geq i$, and $\times^{i} L \lessdot \operatorname{Aut}^{i}(L)$. It follows from Theorem 3.7 that $\times^{i+1} L$ is characteristic in $\operatorname{Aut}^{i}(L)$, and from Lemma 4.3 we have $\operatorname{Rist}_{\mathrm{Aut}^{i+1}(L)}(w) \leq \operatorname{Aut}(L)$ as soon as $|w| \geq i$. Therefore $\operatorname{Rist}_{\mathrm{Aut}^{i+1}(L)}(w x) \leq \operatorname{Rist}_{\mathrm{Aut}(L)}(x) \leq L$ for all $x \in \mathcal{T}$, and $\operatorname{Rist}_{\mathrm{Aut}^{i+1}(L)}(w)=L$ as soon as $|w| \geq i+1$.

Then, again by Theorem 3.7. $\times{ }^{i+1} L \lessdot$ Aut $^{i+1}(L)$, because the product of all rigid stabilisers on a level of the tree is always characteristic.

Lemma 4.10. Let $L$ be a layered group, and set $N=\operatorname{Aut}(L)$. Then $\operatorname{Aut}^{i}(L) \leq$ $N$ ᄀ $\left(i^{i-1} C_{2}\right)$ for all $i \in \mathbb{N}$.

Proof. By Theorem 3.7, the terms $N_{i}$ of the automorphism tower of $L$ are subgroups of $W$. By Lemma 4.9, the rigid stabilisers at level $i$ in $\mathcal{T}$ are all $L$. Given $\alpha \in$ Aut $^{i+1}(L)$, we decompose it $i$ times as $\alpha=\left(\alpha_{u}, \ldots, \alpha_{v}\right) \pi$ with $\alpha_{w} \in W$ for all $w \in \mathcal{T}$ of length $i$, and $\pi \in i^{i} C_{2}$. $N$.

Since $\alpha$ normalizes $\operatorname{Aut}^{i}(L)$, each $\alpha_{u}$ must normalize $\operatorname{Rist}_{\text {Aut }^{i}(L)}(u)=L$, so $\alpha_{u} \in$

Proof of Theorem 1.3. The first part follows directly from Lemma 4.10

For the second part, Theorem 4.4 tells us how to compute $\operatorname{Norm}^{i+1}(L)$ from $\operatorname{Norm}^{i}(L)$. It remains to check that, given Norm ${ }^{i}=\left(\times U_{i}\right)\langle\sigma\rangle\left(\triangle S_{i}\right)$ with $U_{i}, S_{i} \in$ $\mathcal{C}$, we have $U_{i+1}$ and $S_{i+1} \in \mathcal{C}$. This is clealy true for $U_{i+1}$, and for $S_{i+1}$ the computations of Theorem 4.7 show that it holds for $S_{i+1}$ if $i \leq 3$.

Then consider $i \geq 4$, and the group $U_{i} \in \mathcal{C}$. By Lemma 4.9, we may write

$$
U_{i}=\left(\prod_{Z \in\{\times, \triangle\}^{i}} Z G_{Z}\right)\left(i^{i}\langle\sigma\rangle\right),
$$

and we have $G_{\times^{i}}=L$. The recursive computation of Norm $U_{i}$, following Theorem 4.4, asks us to compute $\operatorname{Norm}\left(G_{\times^{i}}\right)=N$ and its intersection with some of the 
$\operatorname{Norm}\left(G_{Z}\right)$; for this last step it suffices to compute $\operatorname{Norm}_{N}\left(G_{Z}\right)$ and take intersections, all operations that leave elements of $\mathcal{C}$.

The same argument applies to $\operatorname{Norm}_{U_{i}}\left(S_{i}\right)=S_{i+1}$.

We next construct within $W$ a family of layered subgroups of arbitrary height $n \in \mathbb{N}$. Set

$$
L_{n}=\left\langle\gamma_{n}(W), F\right\rangle,
$$

where $\gamma_{n}(W)$ is the $n$th term in the lower central series of $W$.

Theorem 4.11. $L_{n}$ is a layered group of height $n-1$; we have Aut ${ }^{m}\left(L_{n}\right)=L_{n-m}$ for all $m \in\{0, \ldots, n-1\}$.

Proof. We note that $L_{n}$ is layered by Lemma 3.3.

We recall the following facts from [4]: the quotient $\gamma_{i}(W) / \gamma_{i+1}(W)$ is isomorphic to the $\mathbb{Z} / 2$-module $\{f: \mathbb{N} \rightarrow \mathbb{Z} / 2\}$, and maps to $\gamma_{i+1}(W) / \gamma_{i+2}(W)$ with finite kernel and co-kernel by commutation with $\dashv^{k} \sigma$, where $2^{k} \| i$.

It suffices to check Norm $L_{n+1}=L_{n}$ for all $n \in \mathbb{N}$. First, $\left[L_{n}, L_{n+1}\right] \leq L_{n+1}$ because $\left[\gamma_{n}(W), F\right] \leq \gamma_{n+1}(W)$ and $\left[\gamma_{n}(W), \gamma_{n+1}(W)\right] \leq \gamma_{n+1}(W)$; therefore Norm $L_{n+1} \geq L_{n}$.

Second, given $g \notin L_{n}$, there exists $i<n$ with $g \in L_{i} \backslash L_{i+1}$. Then by [4] there exists $f \in F$ with $[g, f] \notin L_{i+2}$, so in particular $[g, f] \notin L_{n+1}$ and $g \notin$ Norm $L_{n+1}$.

4.1. The dihedral, affine and cardioid group. Here we compute the automorphism and normalizer tower of three examples; the first two are not layered, while the third one is. We start by fixing some common notation. Consider the adding machine $\tau=(1, \tau) \sigma$ acting on the binary tree $\mathcal{T}=\{1,2\}^{*}$. It generates an infinite cyclic group. Also consider the order-2 isometry $\delta$ of $\mathcal{T}$, defined by $\delta=(\delta, \delta) \sigma$.

Theorem 4.12. The group $H=\langle\tau, \delta\rangle$ is infinite dihedral, and satisfies $\operatorname{Norm}_{W}(H)$ $=H$.

Proof. We have $\delta=u_{-1} \tau^{-1}$, and therefore $\tau^{\delta}=\tau^{-1}$ and $H$ is infinite dihedral.

Then $\operatorname{Aut}(H)$ is also dihedral, generated by $H$ and a new element $v$ satisfying $v^{2}=\tau$ and $v^{\delta}=v^{-1}$. We have $[\operatorname{Aut}(H): H]=2$.

However, there exists no tree isometry $g$ satisfying $g^{2}=\tau$; therefore $\operatorname{Norm}(H)=$ $H$ and the tower of $H$ strictly contains the normalizer tower.

Note that $H$ is a finitely generated solvable group with $\operatorname{Norm}(H)$ countable. In contrast, if $B$ is an abelian or finite 2-subgroup of Aut $\mathcal{T}$, then $\operatorname{Cent}_{\text {Aut }} \mathcal{T}(B)$ is uncountable.

Next consider the topological closure of $\langle\tau\rangle$. It is $T=\left\{\tau^{x} \mid x \in \mathbb{Z}_{2}\right\} \cong \mathbb{Z}_{2}$, the triangular group. Concretely, $\tau^{x}$ can be defined as

$$
\tau^{x}= \begin{cases}\left(\tau^{x / 2}, \tau^{x / 2}\right) & \text { if } x \in 2 \mathbb{Z}_{2}, \\ \left(\tau^{(x-1) / 2}, \tau^{(x+1) / 2}\right) \sigma & \text { if } x \in 2 \mathbb{Z}_{2}+1 .\end{cases}
$$

Also consider the group $U=\left\{u_{x} \mid x \in \mathbb{Z}_{2}^{\bullet}\right\} \cong \mathbb{Z}_{2}^{\bullet}$, the unitary group, where

$$
u_{x}=\left(u_{x}, u_{x} \tau^{(x-1) / 2}\right) \text {. }
$$

Finally set $A=T U$, the affine group. The element $\tau^{x} u_{y}$ can be represented by the matrix $\left(\begin{array}{ll}1 & x \\ 0 & y\end{array}\right)$ over $\mathbb{Z}_{2}$. 
Theorem 4.13. We have

$$
\operatorname{Norm}_{W}(T)=A, \quad \operatorname{Norm}_{W}(A)=A .
$$

Proof. We first note that $\operatorname{Cent}_{W}(T)=T$. If $\alpha \in W$ fixes $\tau$, write $\alpha=\left(\alpha_{1}, \alpha 2\right) \sigma^{i}$. Then $\tau^{\alpha}=\sigma^{i}\left(\alpha_{1}^{-1} \alpha_{2}, \alpha_{2}^{-1} \tau \alpha_{1}\right) \sigma^{1-i}=(1, \tau) \sigma$, so either $i=0, \alpha_{1}=\alpha_{2} \in \operatorname{Cent}_{W}(\tau)$ or $i=1, \alpha_{1}=\alpha_{2} \tau^{-1} \in \operatorname{Cent}_{W}(\tau)$; so in all cases, $\alpha \in T$. Since $T$ has $\langle\tau\rangle$ as a dense subgroup, the claim follows.

Now since $W$ acts continuously on itself by conjugation, any $a \in \operatorname{Norm}_{W}(T)$ is determined by its value on $\tau$, and a simple computation shows that $\tau^{u_{x}}=\tau^{x}$, proving the first equality.

Next, $T$ is a $\operatorname{Norm}_{W}(A)$-invariant subgroup of $A$, since it can be defined as the set of fixed-point-free permutations of $A$; therefore any $a \in \operatorname{Norm}_{W}(A)$ is again determined by its value on $\tau$, and the second equality follows.

However, the automorphism tower of $T$ grows higher: even restricting to continuous automorphisms, we have $\operatorname{Aut}^{n}(A)=\frac{1}{2^{n}} \mathbb{Z}_{2} \rtimes \mathbb{Z}_{2}^{\bullet} \cong A$, Aut ${ }^{\omega}(A)=\mathbb{Q}_{2} \rtimes \mathbb{Z}_{2}^{\bullet}$, and $\operatorname{Aut}^{\omega+1}(A)=\operatorname{Aut}^{\omega+2}(A)=\mathbb{Q}_{2} \rtimes \mathbb{Q}_{2}$.

Our last example is the group $G=\langle F, \tau\rangle$, where $F$ denotes the "finitary group" generated by $\left\{1^{n} * \sigma \mid n \in \mathbb{N}\right\}$. We call $G$ the Cardioid group; its name derives from the "monodromy construction" of V. Nekrashevych [3], when applied to the polynomial $f(z)=z^{2}+\epsilon$, with $\epsilon$ in the Mandelbrot set's main cardioid. We briefly summarize its construction: let $M$ be the Riemann surface $\mathbb{C} \backslash \overline{\left\{f^{n}(\epsilon)\right\}_{n \geq 0}}$, and let $M_{0}=M \backslash\{0\}$ be an open subsurface of $M$. Then $f$ defines a 2-fold covering from $M_{0}$ to $M$. Let $t \in M$ be an arbitrary point, and let $\mathcal{T}=\bigsqcup_{n \geq 0} f^{-n}(t) \times\{n\}$ be the disjoint union of all preimages of $t$. It naturally has the structure of a binary tree, with edges connecting $(x, n+1)$ to $(f(x), n)$. We let $\pi_{1}(M, t)$ act by monodromy (path lifting) on $\mathcal{T}$, and denote by $\operatorname{IMG}(f)$ the quotient of $\pi_{1}(M, t)$ by the kernel of the action.

It can be shown that $G=\operatorname{IMG}(f)$. Indeed $\pi_{1}(M, t)$ is free, and as generators of $\pi_{1}(M, t)$ one may choose a loop around $\infty$, giving $\tau$, and loops encircling $\overline{\left\{f^{m}(\epsilon)\right\}_{m \geq n}}$, giving $1^{n} * \sigma$.

Theorem 4.14. (1) $G$ is a countable layered group, and satisfies $G / G^{\prime}=\mathbb{Z} \oplus(\mathbb{Z} / 2)^{\infty}$.

(2) $\operatorname{Norm}(G)=\langle F, T\rangle D$, where $D=\langle\delta\rangle \cong \mathbb{Z} / 2$.

(3) $\operatorname{Norm}^{2}(G)=\operatorname{Norm}(G)$.

Proof. Clearly $G$ is layered, because $\tau \sigma=(1, \tau) \in G$. The abelianization of $W$ is $(\mathbb{Z} / 2)^{\mathbb{N}}$, and the image of $G$ in $W / W^{\prime}$ is generated by the finite-support and constant functions. Furthermore, the image of $\tau$ in $G / G^{\prime}$ has infinite order, since $\tau^{2^{n}} \in G^{\prime}$ implies $\tau \in G^{\prime}$ contradicting the above.

For the second assertion, we first check that $T$ normalizes $F$; indeed

$$
\sigma^{\tau^{x}}=\left\{\begin{array}{ll}
\left(\tau^{-x / 2}, \tau^{-x / 2}\right) \sigma\left(\tau^{x / 2}, \tau^{x / 2}\right) & \text { if } x \in 2 \mathbb{Z}_{2} \\
\sigma\left(\tau^{(1-x) / 2}, \tau^{(-1-x) / 2}\right) \sigma\left(\tau^{(x-1) / 2}, \tau^{(x+1) / 2}\right) \sigma & \text { if } x \in 2 \mathbb{Z}_{2}+1
\end{array}=\sigma,\right.
$$

and then by induction

$$
\left(1^{n} * \sigma\right)^{\tau^{x}}=\left\{\begin{array}{ll}
\left(\left(1^{n-1} * \sigma\right)^{\tau^{x / 2}}, 1\right) & \text { if } x \in 2 \mathbb{Z}_{2} \\
\left(1,\left(1^{n-1} * \sigma\right)^{\tau^{(x-1) / 2}}\right) & \text { if } x \in 2 \mathbb{Z}_{2}+1
\end{array} \in \sigma^{F} .\right.
$$

Since $T$ clearly centralizes $\tau$, it follows that $T$ normalizes $G$. 
Next, we compute $\operatorname{Norm}(G) \cap A=T D$; indeed $\sigma^{u_{x}}=\left(\tau^{(x-1) / 2},\left(\tau^{(x-1) / 2}\right)^{u_{x}}\right) \sigma$ belongs to $G$, and therefore $(x-1) / 2 \in \mathbb{Z}$, so $x \in 2 \mathbb{Z}+1$ is invertible, and $x= \pm 1$.

Finally, consider an arbitrary $a \in \operatorname{Norm}(G)$. Since $\tau^{a} \in G$, we have $\tau^{a}=$ $\left(\tau^{i_{1 \ldots 1}}, \ldots, \tau^{i_{2 \ldots 2}}\right) \pi$ for some permutation $\pi$ of the first $n$ levels of $\mathcal{T}$. Furthermore $\pi$ is a $2^{n}$-cycle, and therefore $\left(\tau^{2^{n}}\right)^{a}=\left(\tau^{j}, \ldots, \tau^{j}\right)$ for some odd $j \in \mathbb{Z}$.

On the other hand, since $G$ is layered, we may decompose

$$
a=(b, \ldots, b)\left(1, g_{1 \ldots 12}, \ldots, g_{2 \ldots 2}\right) \pi
$$

for some $g_{v} \in G$ and $b \in \operatorname{Norm}(G)$. Then $\tau^{b}=\tau^{j}$, and therefore $b \in T D$, so $a \in F T D$.

The last assertion follows from Theorem 4.7. Indeed in that theorem's notation $U_{2}=\Omega(\operatorname{Norm}(G), G)=G$, since $\left[\tau^{x} d, \tau^{y}\right]=\tau^{2 y} \notin G$ as soon as $y \notin \mathbb{Z}$, whence $U_{2} \leq F T$; and $\left(\tau^{x}\right)^{2} \notin G$ for $x \notin \mathbb{Z}$, whence $U_{2}=F\langle\tau\rangle$. Therefore $\operatorname{Norm}^{2}(G)=$ $\operatorname{Norm}(G)\left(\times U_{2}\right)=\operatorname{Norm}(G)$.

\section{The three Groups $\ddot{\Gamma}, \dot{\Gamma}, \Gamma$ ACTING ON THE TERnARY TREe}

We consider in this section groups acting on the ternary tree $\mathcal{T}=X^{*}$ with $X=\{1,2,3\}$, that are generated by two elements $x$ and $\gamma$. The 3 -cycle $x=(1,2,3)$ induces a rigid permutation of the subtrees $1 \mathcal{T}, 2 \mathcal{T}, 3 \mathcal{T}$ of $\mathcal{T}$ as in (1). Now choose $i, j \in\{0,1,2\}$ and consider the element $\gamma=\left(\gamma, x^{i}, x^{j}\right)$ acting recursively as $\gamma, x^{i}, x^{j}$ on the respective subtrees $1 \mathcal{T}, 2 \mathcal{T}, 3 \mathcal{T}$. Up to conjugation by a tree isometry, it is sufficient to consider such groups with $i=1$, and therefore there are at most three non-isomorphic such groups. These groups are known to be non-isomorphic [2]. We concentrate here on the first example, the group $\ddot{\Gamma}$ for which $\gamma=\left(\gamma, x, x^{-1}\right)$. It was first studied in [16].

Recall that a group is just-infinite if it is infinite, but all its proper quotients are finite. The main properties of $\ddot{\Gamma}$ are:

Theorem 5.1. $\ddot{\Gamma}$ is a just-infinite 3 -group, and it is regular branch: we have $\ddot{\Gamma}^{\prime}>$ $\times \ddot{\Gamma}^{\prime}$ with $\ddot{\Gamma} / \ddot{\Gamma}^{\prime} \cong(\mathbb{Z} / 3) \times(\mathbb{Z} / 3)$ and $\ddot{\Gamma}^{\prime} /\left(\ddot{\Gamma}^{\prime} \times \ddot{\Gamma}^{\prime} \times \ddot{\Gamma}^{\prime}\right) \cong(\mathbb{Z} / 3) \times(\mathbb{Z} / 3)$.

5.1. The original strategy. This section describes the automorphism tower of the group $\ddot{\Gamma}$, its normalizer in the pro-3-Sylow of $W$, and sketches similar results for two related groups acting on the ternary tree. We start by reviewing the strategy followed by the second author in 32 to compute the automorphism group of $\ddot{\Gamma}$ :

(1) Define the ascending series of subgroups $\ddot{\Gamma}^{0}=\ddot{\Gamma}$ and $\ddot{\Gamma}^{n+1}=\ddot{\Gamma}^{n}\langle\langle x\rangle$; all these groups act level-transitively on $\mathcal{T}$. Set $\ddot{\Gamma}_{*}=\bigcup_{n \in \mathbb{N}} \ddot{\Gamma}^{n}$.

(2) Observe that $\operatorname{Rist}_{\ddot{\Gamma}}(1) \imath\langle x\rangle \leq \ddot{\Gamma} \leq \ddot{\Gamma} \imath\langle x\rangle=\ddot{\Gamma}^{1}$. Since $\ddot{\Gamma}^{1} /\left(\operatorname{Rist}_{\ddot{\Gamma}}(1) \succ\langle x\rangle\right)$ is a finite 3 -group, it follows that $\operatorname{Norm}_{\ddot{\Gamma}^{1}}(\ddot{\Gamma}) \geq \ddot{\Gamma} ; \operatorname{check}$ that $\operatorname{Norm}_{\ddot{\Gamma}^{1}}(\ddot{\Gamma})=$ $\ddot{\Gamma}\langle(x, x, x)\rangle$. This procedure is repeated for the pair $\left(\operatorname{Rist}_{\ddot{\Gamma}} \imath^{n}\langle x\rangle, \ddot{\Gamma}^{n}\right)$ for all $n \in \mathbb{N}$, and yields $\operatorname{Norm}_{\ddot{\Gamma}_{*}}(\ddot{\Gamma})$.

(3) Determine 3 -automorphisms of $\ddot{\Gamma}$ : produce a Klein 4-group acting simply on the generating set $\left\{x, x^{-1}, \gamma, \gamma^{-1}\right\}$ of $\ddot{\Gamma}$.

(4) The problem is reduced to the description of the IA-automorphisms of $\ddot{\Gamma}$. Express the IA-automorphisms as (potentially infinite) words in $\operatorname{Norm}_{\ddot{\Gamma}_{*}}(\ddot{\Gamma})$.

\footnotetext{
${ }^{4}$ I.e. those automorphisms which induce the identity on $\ddot{\Gamma} / \ddot{\Gamma}^{\prime}$.
} 
(5) Eliminate the possibility of infinite words (i.e. elements of $\widehat{\operatorname{Norm}_{\Gamma_{*}}}(\ddot{\Gamma})$ ) by using induction on the depth function of $\ddot{\Gamma}$, defined on $g$ as the minimal level $n$ at which all states $g @ v$ belong to $\left\{1, x^{ \pm 1}, \gamma^{ \pm 1}\right\}$ for all $v \in X^{n}$.

Theorem 5.2 ([32]). Let $t$ be the transposition $(2,3)$. Set $U=\left\langle\triangle^{n}(x): n \geq 1\right\rangle$, a countably infinite elementary abelian 3-group, and $V=\left\{1, \tau_{1}, \tau_{2}, \tau_{3}\right\}$, a Klein group, where

Then we have a split extension

$$
\tau_{1}=\triangle\left(\tau_{1}\right) t, \quad \tau_{2}=\triangle\left(\tau_{3}\right), \quad \tau_{3}=\triangle\left(\tau_{2}\right) t .
$$

$$
\operatorname{Aut}(\ddot{\Gamma})=\ddot{\Gamma}: U: V .
$$

Furthermore $V$ normalizes $U$, and acts on it via

$$
\begin{aligned}
& \triangle^{n}(x)^{\tau_{1}}=\triangle^{n}(x)^{-1}, \quad \triangle^{n}(x)^{\tau_{2}}=\triangle^{n}(x)^{(-1)^{n}}, \quad \triangle^{n}(x)^{\tau_{3}}=\triangle^{n}(x)^{-(-1)^{n}}, \\
& \gamma^{\tau_{1}}=\gamma, \quad \gamma^{\tau_{2}}=\gamma^{-1}, \quad \gamma^{\tau_{3}}=\gamma^{-1} .
\end{aligned}
$$

Theorem 5.3. Set $T=\left\langle\nabla^{2 n+1}(x): n \geq 0\right\rangle$, a countably infinite elementary abelian 3-group. Then

$$
\operatorname{Aut}^{2}(\ddot{\Gamma})=\operatorname{Aut}(\ddot{\Gamma}): T .
$$

Furthermore $T$ centralizes $U$ and $V$

$$
\operatorname{Aut}^{3}(\ddot{\Gamma})=\operatorname{Aut}^{2}(\ddot{\Gamma}) .
$$

Remark 5.4. It follows from the proof that $\ddot{\Gamma}^{\prime}$ is absolutely characteristic in $\ddot{\Gamma}$, and $\operatorname{Aut}^{2}(\ddot{\Gamma})$ is a subgroup of the commensurator of $\ddot{\Gamma}$.

To simplify the notations, we write $A=\operatorname{Aut}(\ddot{\Gamma})$ and $B=\operatorname{Aut}^{2}(\ddot{\Gamma})$. For $v \in X^{n}$, we also write

$$
\operatorname{Stab}_{\ddot{\Gamma}}^{v}(n)=\left\{g \in \operatorname{Stab}_{\ddot{\Gamma}}(n) \mid g @ v=1\right\} .
$$

Lemma $5.5\left([32) . \ddot{\Gamma}^{\prime}\right.$ is a subdirect product of $\ddot{\Gamma} \times \ddot{\Gamma} \times \ddot{\Gamma}$, and factors as

$$
\ddot{\Gamma}^{\prime}=\left(\ddot{\Gamma}^{\prime} \times \ddot{\Gamma}^{\prime} \times \ddot{\Gamma}^{\prime}\right)\langle[\gamma, x], \triangle \gamma\rangle .
$$

Furthermore, $\operatorname{Cent}_{W}\left(\ddot{\Gamma}^{\prime}\right)$ is trivial.

Lemma 5.6. $A^{\prime}=\ddot{\Gamma}: U$, and $A^{\prime \prime}=\ddot{\Gamma}^{\prime}$. Therefore $\ddot{\Gamma}^{\prime}$ is characteristic in $A$.

Proof. The first assertion follows immediately from the fact that the every element of the generating set $\left\{\gamma, \triangle^{n}(x): n \geq 0\right\}$ of $\ddot{\Gamma} U$ is inverted by $\tau_{1}$ or $\tau_{2}$. The second assertion follows from $A^{\prime \prime}=\ddot{\Gamma}^{\prime}[\ddot{\Gamma}, U]$, and $\left[x, \triangle^{n}(x)\right]=1,\left[\gamma, \triangle^{n}(x)\right]=$ $\left(\left[\gamma, \triangle^{n-1}(x)\right], 1,1\right) \in \ddot{\Gamma}^{\prime}$.

Lemma 5.7. $B \leq(A \times A \times A)\left\langle x, \tau_{1}\right\rangle$.

Proof. First, note that $A$ is saturated (the subgroups $\left\langle v * \ddot{\Gamma}^{\prime}:|v|=n\right\rangle$ may serve as $H_{n}$ ) and weakly branch (since it contains $\ddot{\Gamma}$ ), and apply Theorem 3.7 to obtain $B \cong \operatorname{Norm}_{W}(A)$; these two groups will be identified. As $\ddot{\Gamma}^{\prime}$ is $B$-invariant and Cent $_{W}\left(\ddot{\Gamma}^{\prime}\right)=\{e\}$, we conclude that $B \leq \operatorname{Aut}\left(\ddot{\Gamma}^{\prime}\right)$.

Given $\phi \in B$, it can be written as $\phi=\left(\phi_{1}, \phi_{2}, \phi_{3}\right) \tau_{1}^{i} x^{j}$ for some $i \in\{0,1\}$ and $j \in\{0,1,2\}$. We conclude that $\left(\phi_{1}, \phi_{2}, \phi_{3}\right) \in B$. Now since $\ddot{\Gamma}^{\prime}$ is a subdirect product of $\ddot{\Gamma} \times \ddot{\Gamma} \times \ddot{\Gamma}$, we obtain $\phi_{x} \in \operatorname{Norm}_{W}(\ddot{\Gamma})$ for $x \in X$. The conclusion follows. 
Lemma 5.8 ([32]). Take $g \in \ddot{\Gamma}$. Then $g \in \operatorname{Stab}_{\ddot{\Gamma}}(1)$ if and only if

$$
g=\left(c_{1} \gamma^{n_{1}} x^{n_{3}-n_{2}}, c_{2} \gamma^{n_{2}} x^{n_{1}-n_{3}}, c_{3} \gamma^{n_{3}} x^{n_{2}-n_{1}}\right)
$$

for some $c_{x} \in \ddot{\Gamma}^{\prime}$ and $n_{1}, n_{2}, n_{3} \in\{0,1,2\}$. We have

$$
\begin{aligned}
\operatorname{Stab}_{\ddot{\Gamma}}(1) & =\left(\ddot{\Gamma}^{\prime} \times \ddot{\Gamma}^{\prime} \times \ddot{\Gamma}^{\prime}\right)\left\langle\triangle \gamma,\left(1, \gamma x^{-1}, \gamma x\right),\left(x, x^{-1}, \gamma\right)\right\rangle \\
\text { and } \operatorname{Stab}_{\ddot{\Gamma}}^{1}(1) & =\left(1 \times \ddot{\Gamma}^{\prime} \times \ddot{\Gamma}^{\prime}\right)\left\langle\left(1, \gamma x^{-1}, \gamma x\right) .\right.
\end{aligned}
$$

Lemma 5.9.

$$
\begin{aligned}
\operatorname{Stab}_{A}(1) & =\operatorname{Stab}_{\ddot{\Gamma}}(1) U\left\langle\tau_{2}\right\rangle \\
\text { and } \operatorname{Stab}_{A}^{1}(1) & =\operatorname{Stab}_{\ddot{\Gamma}}^{1}(1)\left\langle\left(1, \gamma^{-1}, \gamma\right)\right\rangle,
\end{aligned}
$$

which is a subdirect product of $1 \times \ddot{\Gamma} \times \ddot{\Gamma}$.

Proof. The first statement is clear. Then take $\phi \in \operatorname{Stab}_{A}^{1}(1)$; we can write

$$
\phi=\left(c_{1} \gamma^{n_{1}} x^{n_{3}-n_{2}} u v, c_{2} \gamma^{n_{2}} x^{n_{1}-n_{3}} u v, c_{3} \gamma^{n_{3}} x^{n_{2}-n_{1}} u v\right)
$$

for some $u \in U @ 1=U\langle x\rangle$ and $v \in V$. Since $c_{1} \gamma^{n_{1}} x^{n_{3}-n_{2}} u v=1$, we have $n_{1}=$ $0, c_{1}=1, v=1$, and $u \in\langle x\rangle$ with $x^{n_{3}-n_{2}} u=1$. Modulo Stab $b_{\tilde{\Gamma}}^{1}(1)$, it suffices to consider $u=x$ and $n_{2}=2, n_{3}=1$, leading to $\left(1, \gamma^{-1}, \gamma\right)$.

The last assertion follows, since the second and third coordinates of $\left\langle\left(1, \gamma x^{-1}, \gamma x\right)\right.$, $\left.\left(1, \gamma^{-1}, \gamma\right)\right\rangle$ each equal $\ddot{\Gamma}$.

Lemma 5.10. Let $H$ be a group such that $A \leq H \leq(A \times A \times A)\langle x\rangle V$, and take $\phi \in H$. Then $\phi$ can be reduced modulo $A$ to $\left(v_{1}, u_{2} v_{2}, x^{i} \gamma^{j} u_{3} v_{3}\right)$.

Proof. Direct.

Lemma 5.11. Consider $\phi=\nabla^{2 n+1}(x)$, for $n \geq 0$. Then $\phi \in B$ and $[U V, \phi]=1$.

Proof. To show that $\phi \in B$ it suffices to show $[g, \phi] \in A$ for all generators $g$ of $A$. First, $[x, \phi]=\triangle^{2 n+2}(x)$ and $[\gamma, \phi]=\left[\triangle^{m}(x), \phi\right]=1$. Then we have

$$
\begin{aligned}
{\left[\tau_{1}, \phi\right] } & \left.=\nabla^{2 n+1}(x)^{-\tau_{1}} \phi=\nabla \triangle^{2 n+1}(x)^{\tau_{1}}\right) \phi=\nabla^{2 n+1}(x)^{-1} \phi=1 \\
\text { and }\left[\tau_{2}, \phi\right] & \left.=\nabla^{2 n+1}(x)^{-\tau_{2}} \phi=\nabla \triangle^{2 n+1}(x)^{-\tau_{3}}\right) \phi=\nabla^{2 n+1}(x)^{-1} \phi=1,
\end{aligned}
$$

since $\tau_{1}$ inverts $\triangle^{2 n+1}(x)$ and $\tau_{2}$ fixes $\triangle^{2 n+1}(x)$.

Proof of Theorem 5.3, first part. Take $\phi$ normalizing $A$, and write it, using Lemma 5.10, as $\phi=\left(v_{1}, u_{2} v_{2}, x^{i} \gamma^{j} u_{3} v_{3}\right)$. We compute the commutator of $\phi$ with generators of $A$ in turn:

\section{interaction with $\tau_{2}$ :}

$$
\begin{aligned}
\phi^{\tau_{2}} & =\left(v_{1}, u_{2} v_{2}, x^{i} \gamma^{j} u_{3} v_{3}\right)^{\triangle \tau_{3}}=\left(v_{1}, u_{2}^{\tau_{3}} v_{2}, x^{-i} \gamma^{-j} u_{3}^{\tau_{3}} v_{3}\right), \\
{\left[\tau_{2}, \phi^{-1}\right] } & =\left(1,\left[\tau_{3}, u_{2}^{-1}\right], x^{-i} \gamma^{-j}\left[\tau_{3}, u_{3}^{-1}\right] \gamma^{-j} x^{-i}\right) \in \operatorname{Stab}_{A}^{1}(1) .
\end{aligned}
$$

Therefore $\left[\tau_{3}, u_{2}^{-1}\right]=\left[\tau_{3}, u_{3}^{-1}\right]=e$ and $i=j=0$; hence $\phi=\left(v_{1}, u_{2} v_{2}, u_{3} v_{3}\right)$, where $u_{2}, u_{3} \in \operatorname{Cent}\left(\tau_{3}\right)$; that is, are products of $\triangle^{2 n+1}(x)$. Now using $T$ we may reduce $\phi$ further to the form $\phi=\left(v_{1}, v_{2}, u v_{3}\right)$, for some $u \in U$ a product of $\triangle^{2 n+1}(x)$. 
interaction with $\tau_{1}$ :

$$
\begin{aligned}
\phi^{\tau_{1}} & =\left(v_{1}, v_{2}, u v_{3}\right)^{\triangle\left(\tau_{1}\right) t}=\left(v_{1}, u v_{3}, v_{2}\right)^{\triangle \tau_{1}}=\left(v_{1}, u^{-1} v_{3}, v_{2}\right), \\
{\left[\tau_{1}, \phi^{-1}\right] } & =\left(v_{1}, u^{-1} v_{3}, v_{2}\right)\left(v_{1}, v_{2},\left(u v_{3}\right)^{-1}\right)=\left(1, u^{-1} v_{3} v_{2}, v_{2} v_{3} u^{-1}\right) \in \operatorname{Stab}_{A}^{1}(1) .
\end{aligned}
$$

Therefore $u=1, v_{2}=v_{3}$, and $\phi$ is reduced to the form $\phi=\left(v_{1}, v_{2}, v_{2}\right)$.

\section{interaction with $x$ :}

$$
\left[x, \phi^{-1}\right]^{x}=\left(1, v_{2} v_{1}, v_{1} v_{2}\right) \in \operatorname{Stab}_{A}^{1}(1) .
$$

Therefore $v_{1}=v_{2}$, and $\phi$ is reduced to $\phi=(v, v, v)$. If $v=\tau_{3}$ we have $\phi=\tau_{2} \in A$, so it suffices to consider $\phi=\triangle \tau_{1}$.

\section{interaction with $\gamma$ :}

$$
\left[\triangle \tau_{1}, \gamma^{-1}\right]=\left(\gamma, x^{-1}, x\right)\left(\gamma, x, x^{-1}\right)^{-1}=(1, x, x),
$$

but this last element is not in $\operatorname{Stab}_{A}^{1}(1)$; therefore, this last case is impossible, and these successive steps have reduced $\phi$ to 1 .

Lemma 5.12. $B^{\prime}=\ddot{\Gamma}: U=A^{\prime}$, and $B^{\prime \prime}=\ddot{\Gamma}^{\prime}=A^{\prime \prime}$.

$\operatorname{Stab}_{B}^{1}(1)=\operatorname{Stab}_{A}^{1}(1): T$.

$B$ is a saturated subgroup of $W$, and Aut $B \leq(A \times A \times A)\left\langle x, \tau_{1}\right\rangle$.

Proof. Direct.

Proof of Theorem [5.3, second part. Let $\phi$ normalize B. As before, we may assume $\phi=\left(v_{1}, u_{2} v_{2}, x^{i} \gamma^{j} u_{3} v_{3}\right)$. Now,

$$
\left[\tau_{2}, \phi^{-1}\right]=\left(1,\left[\tau_{3}, u_{2}^{-1}\right], x^{-i} \gamma^{-j}\left[\tau_{3}, u_{3}^{-1}\right] \gamma^{-j} x^{-i}\right) \in \operatorname{Stab}_{B}^{1}(1) .
$$

Therefore $i=j=0$, and $\left(1,\left[\tau_{3}, u_{2}^{-1}\right],\left[\tau_{3}, u_{3}^{-1}\right]\right) \in T$. On one hand, $T$ is centralized by $\tau_{3}$, but on the other hand $\left[\tau_{3}, u_{2}^{-1}\right]$ and $\left[\tau_{3}, u_{3}^{-1}\right]$ are inverted by $\tau_{3}$, since $\tau_{3}$ is an involution. Since $T$ has no 2-torsion, it follows that $\left[\tau_{3}, u_{2}^{-1}\right]=\left[\tau_{3}, u_{3}^{-1}\right]=$ 1. We then have $\phi=\left(v_{1}, u_{2} v_{2}, u_{3} v_{3}\right)$, where $u_{2}, u_{3}$ are centralized by $\tau_{3}$. Using $\left(1, u_{2}, u_{2}^{-1}\right) \in T$, we may reduce to the case $\phi=\left(v_{1}, v_{2}, u v_{3}\right)$.

We next compute

$$
\begin{aligned}
\phi^{\tau_{1}} & =\left(v_{1},\left(u v_{3}\right)^{\tau_{1}}, v_{2}\right)=\left(v_{1}, u^{-1} v_{3}, v_{2}\right), \\
{\left[\tau_{1}, \phi^{-1}\right] } & =\left(1, u^{-1} v_{3} v_{2}, v_{2} v_{3} u^{-1}\right) \in \operatorname{Stab}_{B}^{1}(1) .
\end{aligned}
$$

Therefore $v_{2}=v_{3}$ and $\left[\tau_{1}, \phi^{-1}\right]=\left(1, u^{-1}, u^{-1}\right) \in U T$, hence $u=1$ and the proof is finished.

5.2. The normalizer tower. The reason the automorphism tower of $\ddot{\Gamma}$ stops after 2 steps can be attributed to the involutions in $V$; in the same way that the lower central series of Aut $(\ddot{\Gamma})$ stabilizes at $\ddot{\Gamma}: U$ and that of $\operatorname{Aut}^{2}(\ddot{\Gamma})$ stabilizes at $\ddot{\Gamma}: U T$, the normalizer tower may not be extended beyond $V$.

However, since $\ddot{\Gamma}$ is a subgroup of the pro-3-Sylow $C=\widehat{\langle x\rangle}$ * of $W$, we may consider its normalizer tower within $C$. For this purpose, consider the set $\Omega=$ $\left\{Z x \mid Z \in\left\{\triangle, \nabla^{\dashv}\right\}^{*}\right\}$, with the notation of Definition 3.2. The set $\Omega$ will serve as a basis for the quotients of successive terms of the normalizer tower of $\ddot{\Gamma}$. Define a 
rank function on $\Omega$ by

$$
\begin{aligned}
\operatorname{rank} x & =1, & \operatorname{rank}(\dashv x) & =3 \operatorname{rank} x, \\
\operatorname{rank}(\nabla) & =3 \operatorname{rank} x-1, & \operatorname{rank}(\triangle x) & =3 \operatorname{rank} x-2,
\end{aligned}
$$

\begin{tabular}{|c|c|c|c|c|c|c|c|}
\hline$n k$ & & men & & & & & \\
\hline 1 & $x$ & $\triangle x$ & $\triangle \triangle x$ & $\triangle \triangle \triangle x$ & $\triangle \triangle \triangle \triangle x$ & $\cdots$ & $\triangle^{n} x$ \\
\hline 2 & & $\nabla$ & $\nabla x$ & $\nabla \Delta x$ & $\nabla \triangle \triangle x$ & $\ldots$ & $\nabla^{n} x$ \\
\hline 3 & & $\dashv x$ & $\dashv \triangle x$ & $\dashv \Delta \Delta x$ & $\dashv \Delta \Delta \triangle \Delta x$ & $\ldots$ & $\dashv \Delta^{n} x$ \\
\hline 4 & & & $\Delta \nabla$ & $\Delta \nabla x$ & $\triangle \nabla \triangle x$ & $\ldots$ & $\Delta \nabla^{n} x$ \\
\hline 5 & & & $\nabla x$ & $\nabla \Delta x$ & $\nabla \triangle \triangle x$ & $\ldots$ & $\nabla \triangle^{n} x$ \\
\hline 6 & & & $\dashv \nabla$ & $\dashv \nabla x$ & $\dashv \nabla \Delta x$ & $\ldots$ & $\dashv \nabla^{n} x$ \\
\hline 7 & & & $\triangle \dashv x$ & $\triangle \dashv \triangle x$ & $\triangle \dashv \Delta \triangle x$ & $\ldots$ & $\triangle \dashv \triangle^{n} x$ \\
\hline 8 & & & $\nabla x$ & $\nabla \triangle x$ & $\nabla \triangle \triangle x$ & $\ldots$ & $\nabla \Delta^{n} x$ \\
\hline 9 & & & $\dashv \dashv x$ & $\dashv \dashv \triangle x$ & $\dashv \dashv \triangle \triangle \triangle x$ & $\ldots$ & $\dashv \dashv \triangle^{n} x$ \\
\hline 10 & & & & $\triangle \triangle \nabla$ & $\triangle \triangle \nabla x$ & $\ldots$ & $\triangle \triangle \nabla^{n} x$ \\
\hline
\end{tabular}

and define $A_{n}=\langle x, \gamma, Z x$ such that $\operatorname{rank}(Z x) \leq n\rangle$. The elements of $\Omega$ of low rank are as given below:

The next rows follow the same pattern: elements of rank $n$ are determined by writing backwards $n-1$ in base 3 , using the symbols $\Delta=0, \nabla 1$ and $\dashv=2$.

Theorem 5.13. The following facts hold for all $n \geq 1$ :

(1) $\operatorname{Norm}_{C}^{n}(\ddot{\Gamma}) \geq A_{n}$;

(2) each $\operatorname{Norm}_{C}^{n+1}(\ddot{\Gamma}) / \operatorname{Norm}_{C}^{n}(\ddot{\Gamma})$ is an infinite countable elementary abelian 3group;

(3) $\operatorname{Norm}_{C}^{\alpha}(\ddot{\Gamma})=\langle\gamma, \Omega\rangle=\ddot{\Gamma}_{*}$ for $\alpha \geq \omega$.

Proof. Extend the rank function to $\Omega \cup\{\gamma\}$, by $\operatorname{rank} \gamma=0$. Inductive computations then show that $\operatorname{rank}[u, v] \leq \max \{\operatorname{rank} u, \operatorname{rank} v\}-1$ for all $u \in \Omega \cup\{\gamma\}$ and $v \in \Omega$ of rank at least 1. For example, for any $Y, Z \in\{\triangle, \nabla \dashv\}^{*}$,

$$
\begin{aligned}
\operatorname{rank}[\nabla x, \triangle Z x] & =\operatorname{rank} \nabla][Y x, Z x] \\
& \leq 3 \operatorname{rank}[Y x, Z x]-1 \\
& \leq 3(\max \{\operatorname{rank}(Y x), \operatorname{rank}(Z x)\}-1)-1 \\
& \leq \operatorname{rank} \max \{\operatorname{rank}(\nabla x), \operatorname{rank}(\nabla x)\}-1, \\
\text { and } \operatorname{rank}[x, \nabla x] & =\operatorname{rank} \triangle Z x=\operatorname{rank}(\nabla x)-1 .
\end{aligned}
$$

We therefore have $A_{n} \triangleleft A_{n+1}$ with abelian quotients, so the tower $A_{n}$ grows more slowly than the normalizer tower.

Finally, the tower of $\ddot{\Gamma}$ stops at level $\omega$ by Theorem 3.15. 


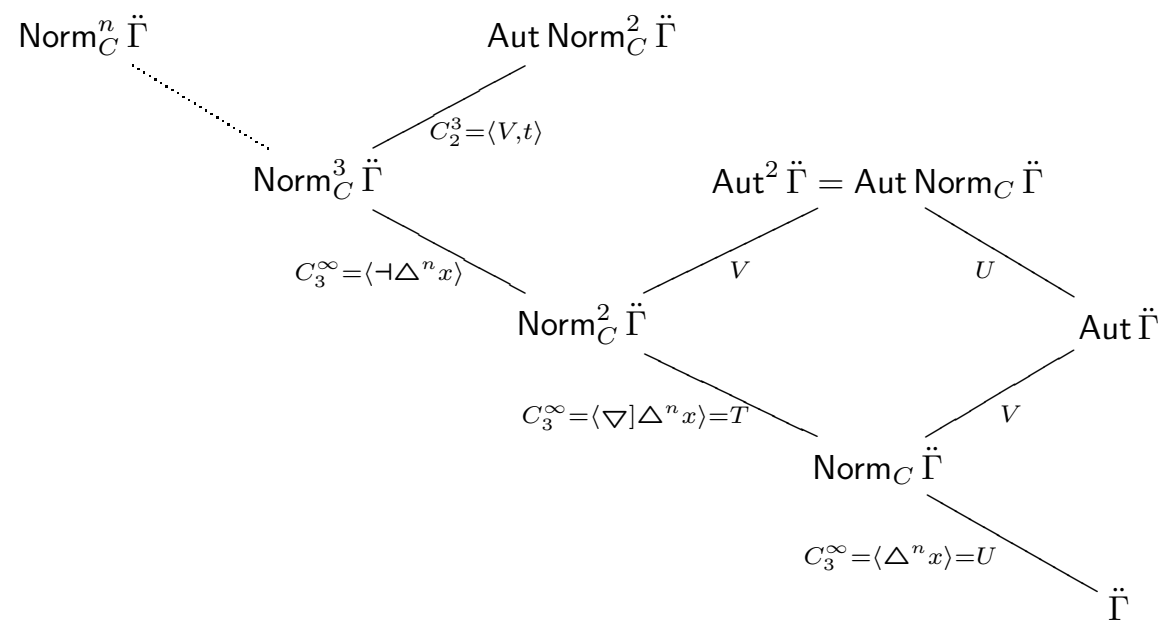

5.3. The Fabrykowski-Gupta group. This group was introduced in [10] as an alternate construction of a group of intermediate growth. Like $\ddot{\Gamma}$, it acts on the ternary tree $\{1,2,3\}^{*}$. Let $x=(1,2,3)$ be the 3 -cycle acting on $\mathcal{T}$ according to (1), and define recursively $\gamma=(\gamma, x, 1)$. The Fabrykowski-Gupta group is $\Gamma=\langle x, \gamma\rangle$. It is a regular branch group; we have $\Gamma / \Gamma^{\prime} \cong(\mathbb{Z} / 3) \times(\mathbb{Z} / 3)$, and $\Gamma^{\prime} /\left(\Gamma^{\prime} \times \Gamma^{\prime} \times \Gamma^{\prime}\right) \cong$ $(\mathbb{Z} / 3) \times(\mathbb{Z} / 3)$.

The generators $x, \gamma$ of $\Gamma$ have order 3 , and $\langle x \gamma, \gamma x\rangle$ is a torsion-free normal subgroup of $\Gamma$ of index 3.

Theorem 5.14. Set $U=\left\langle\triangle^{n}(x): n \geq 1\right\rangle$, a countably infinite elementary abelian 3 -group. Then we have a split extension

$$
\operatorname{Aut}(\Gamma)=\Gamma: U \text {. }
$$

Proof. The proof follows the same scheme as that of Theorem 5.3 and is omitted.

5.4. The group $\dot{\Gamma}$. This group also acts on the ternary tree, and is generated by $x=(1,2,3)$ and $\gamma=(\gamma, x, x)$. The group $\dot{\Gamma}$ was first studied in [1, 2], where some of its elementary properties were proven.

Note that the group $\dot{\Gamma}$ is not branch, but is a regular weakly branch group. Consider the subgroup $K=\left\langle x^{-1} \gamma, \gamma x^{-1}\right\rangle$; then $K$ is a torsion-free normal subgroup of index 3 . We have $\dot{\Gamma} / \dot{\Gamma}^{\prime} \cong(\mathbb{Z} / 3) \times(\mathbb{Z} / 3), \dot{\Gamma}^{\prime} / K^{\prime} \cong \mathbb{Z}^{2}$, and $K^{\prime} /\left(K^{\prime} \times K^{\prime} \times K^{\prime}\right) \cong \mathbb{Z}^{2}$.

Theorem 5.15. Let $t$ be the transposition $(2,3)$ acting on $\mathcal{T}$ according to (11). Let $U=\left\langle\triangle^{n}\left(x \gamma^{-1}\right): n \geq 1\right\rangle$ be a countably infinite elementary abelian 3-group, and let $V=\left\{1, \tau_{1}, \tau_{2}, \tau_{3}\right\}$ be the Klein group defined by

$$
\tau_{1}=\triangle\left(\tau_{1}\right) t, \quad \tau_{2}=\triangle\left(\tau_{1}\right), \quad \tau_{3}=t .
$$

Then we have a non-split extension

$$
\operatorname{Aut}(\dot{\Gamma})=(\dot{\Gamma} \cdot U): V,
$$

where $V$ acts on $U$, for $n>0$, by

$$
x^{\tau_{1}}=x^{-1}, \quad x^{\tau_{2}}=x, \quad x^{\tau_{3}}=x^{-1},
$$




$$
\begin{aligned}
& \triangle^{n}(x)^{\tau_{1}}=\triangle^{n}(x)^{-1}, \quad \triangle^{n}(x)^{\tau_{2}}=\triangle^{n}(x)^{-1}, \quad \triangle^{n}(x)^{\tau_{3}}=\triangle^{n}(x), \\
& \gamma^{\tau_{1}}=\gamma^{-1}, \quad \gamma^{\tau_{2}}=\gamma^{-1}, \quad \gamma^{\tau_{3}}=\gamma .
\end{aligned}
$$

Let $T=\left\langle\nabla^{2 n+1}(x): n \geq 0\right\rangle$ be a countably infinite elementary abelian 3-group. Then

$$
\operatorname{Aut}^{3}(\dot{\Gamma})=\operatorname{Aut}^{2}(\dot{\Gamma})=\operatorname{Aut}(\dot{\Gamma}): T \text {. }
$$

Furthermore $T$ centralizes $U$ and $V$.

Proof. The proof follows the same scheme as that of Theorem 5.3 and is omitted.

\section{The First GrigorChuK GROUP}

This section describes an automorphism tower of the first Grigorchuk group. Consider the regular binary tree $X^{*}$ with $X=\{1,2\}$, denote by $\sigma$ the non-trivial permutation $(1,2)$ of $X$ and define isometries $a, b, c, d$ of $\mathcal{T}$ by

$$
\begin{aligned}
\left(x_{1} \ldots x_{m}\right)^{a} & =x_{1}^{\sigma} x_{2} \ldots x_{m}, \\
b & =(a, c)=\prod_{i \neq 0} 2_{\bmod 3} 2^{i} 1 * a, \\
c & =(a, d)=\prod_{i \neq 2} 2_{\bmod 3}^{i} 1 * a, \\
d & =(1, b)=\prod_{i \neq 1} 2_{\bmod 3}^{i} 1 * a .
\end{aligned}
$$

The Grigorchuk group 13, 14] is $\mathfrak{G}=\langle S\rangle$, with $S=\{a, b, c, d\}$. It is a regular branch group. Set $B=\operatorname{Rist}_{\mathfrak{G}}(1) @ 1=\langle b\rangle^{G}$ and $K=\operatorname{Rist}_{\mathfrak{G}}\left(1^{2}\right) @ 1^{2}=\langle[a, b]\rangle^{\mathfrak{G}}$. Then $B$ has index 8 in $\mathfrak{G}$, and $K$ has index 16 ; and $K /(K \times K) \cong \mathbb{Z} / 4$.

The automorphism group of $\mathfrak{G}$ was computed in [15], where it was shown, in a manner similar to the one employed in 32 , that Out $(\mathfrak{G})$ is an infinite elementary 2-group, generated by $\Delta^{n} \dashv$ adad for all $n \geq 1$, in the notation of Definition 3.2. Below we will give a complete description of the tower, without relying on the above result. In order to illustrate our method, we first examine more closely the first terms of the automorphism tower.

Let us inductively define the normalizer tower by

$$
N_{0}=\mathfrak{G} \quad \text { and } \quad N_{n+1}=\operatorname{Norm}_{W}\left(N_{n}\right) .
$$

Then $N_{1}=\left\langle a, b, c, d, \triangle^{n} \dashv a d a d\right\rangle$. There are clearly some elements of $W$ outside $N_{1}$ that normalize $N_{1}$; for instance, $\dashv a d$. In fact, a little computation shows that the elements $\dashv \triangle^{n} \dashv$ adad for all $n \in \mathbb{N}$ also normalize $N_{1}$ : indeed for all $m, n \geq 0$,

$$
\begin{aligned}
{\left[\dashv \triangle^{n} \dashv a d a d, \triangle^{m} \dashv a d a d\right] } & = \begin{cases}\dashv \triangle^{m-1} \dashv\left[\triangle^{n-m-1} \dashv a d a d, \triangle b\right]=1 & \text { if } m<n+1 ; \\
1 & \text { if } m=n+1, \\
\dashv \triangle^{n} \dashv\left[\triangle b, \triangle^{m-n-2} \dashv a d a d\right]=1 & \text { if } m>n+1 ;\end{cases} \\
{\left[\dashv \triangle^{n} \dashv a d a d, a\right] } & =\triangle^{n+1} \dashv a d a d \in N_{1} ;
\end{aligned}
$$

Similarly, we have $\dashv$ ad in $N_{2}$.

Proceeding, we see $\dashv a$ in $N_{3}$, and therefore $N_{3}$ contains $\times \mathfrak{G}$. We may also check that $N_{3}$ contains some other elements, like $\triangle \dashv a d$ and $\triangle \dashv \dashv$ adad. Based on the 
behaviour prescribed by Theorem 4.7 for layered groups, we may "guess" that $N_{3}$ is $(\times \mathfrak{G})\left(\triangle N_{1}\right)\langle a\rangle$.

Actually, the tower of automorphisms of $\mathfrak{G}$ follows a pattern that is best described by powers of 2 . We describe it in the same language as the normalizer tower of $\ddot{\Gamma}$ given in Subsection 5.2 .

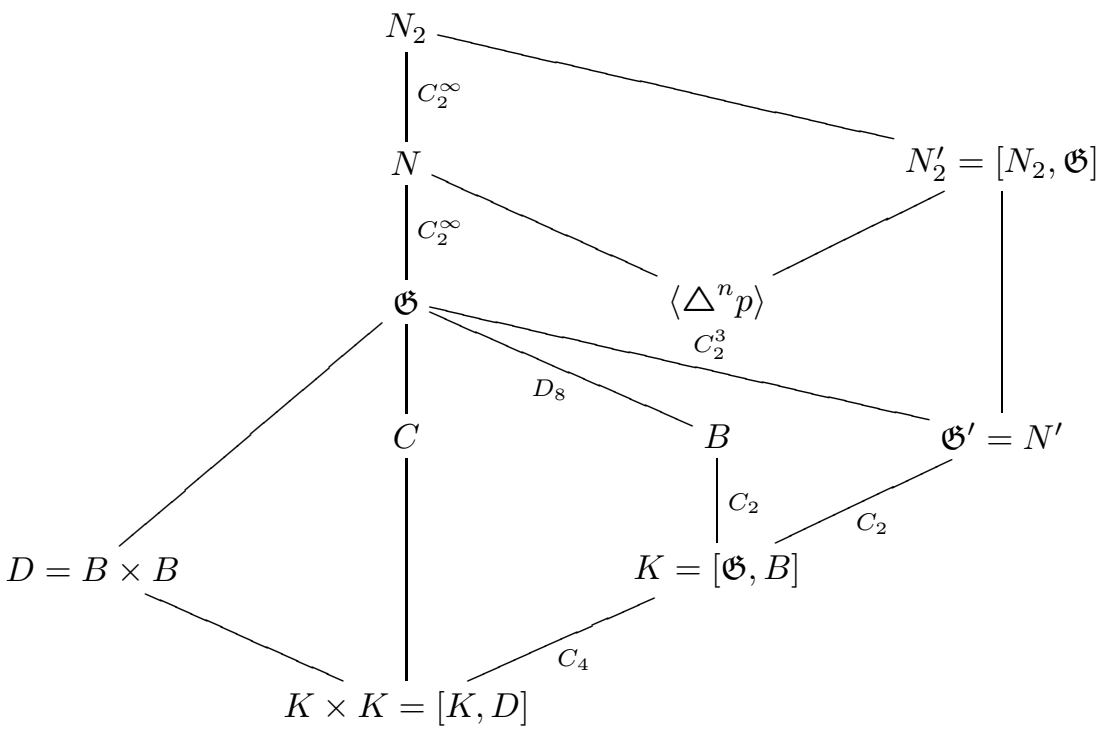

Let us define

$$
p=\dashv a d a d, \quad q=\dashv a d, \quad r=\dashv a,
$$

and consider the subset $\Omega=\left\{Z p, Z q, Z r \mid Z \in\{\triangle, \dashv\}^{*}\right\}$ of $\mathfrak{G}_{*}$, which will serve as a basis for the quotients of successive terms of the automorphism tower of $\mathfrak{G}$. Define a rank on $\Omega$ by

$$
\operatorname{rank} p=1, \quad \operatorname{rank} q=2, \quad \operatorname{rank} r=3
$$

and inductively

$$
\operatorname{rank}(\dashv x)=2 \operatorname{rank} x, \quad \operatorname{rank}(\triangle x)=2 \operatorname{rank} x-1 .
$$

Then $\langle\Omega \cup S\rangle=\mathfrak{G}_{*}$ is the layered closure of $\mathfrak{G}$. Extend the rank function to $\Omega \cup S$ by $\operatorname{rank}(s)=0$ for all $s \in S$, and define the following subgroups of $\mathfrak{G}_{*}$ :

\begin{tabular}{|c|c|c|c|c|c|c|c|c|c|}
\hline ink & & & & & & & & & \\
\hline 1 & & & $p$ & $\triangle p$ & $\triangle \triangle p$ & $\triangle \triangle \triangle p$ & $\triangle \triangle \triangle \triangle p$ & $\cdots$ & $\Delta^{n} p$ \\
\hline 2 & & $q$ & & $\dashv p$ & $\dashv \triangle p$ & $\dashv \triangle \triangle p$ & $\dashv \triangle \Delta \triangle p$ & $\ldots$ & $\dashv \triangle^{n} p$ \\
\hline 3 & $r$ & $\triangle q$ & & & $\Delta \dashv p$ & $\triangle \dashv \triangle p$ & $\triangle \dashv \triangle \triangle p$ & $\ldots$ & $\triangle \dashv \triangle^{n} p$ \\
\hline 4 & & $\dashv q$ & & & $\dashv \dashv p$ & $\dashv \dashv \triangle p$ & $\dashv \dashv \Delta \Delta p$ & $\ldots$ & $\dashv \dashv \triangle^{n} p$ \\
\hline 5 & $\Delta r$ & $\triangle \triangle q$ & & & & $\triangle \triangle \dashv p$ & $\triangle \triangle \dashv \triangle p$ & $\ldots$ & $\triangle \triangle \dashv \triangle^{n} p$ \\
\hline 6 & $\dashv r$ & $\dashv \triangle q$ & & & & $\dashv \triangle \dashv p$ & $\dashv \triangle \dashv \Delta p$ & $\ldots$ & $\dashv \triangle \dashv \triangle^{n} p$ \\
\hline 7 & & $\Delta \dashv q$ & & & & $\Delta \dashv \dashv p$ & $\Delta \dashv \dashv \Delta p$ & $\ldots$ & $\triangle \dashv \dashv \triangle^{n} p$ \\
\hline 8 & & $\dashv \dashv q$ & & & & $\dashv \dashv \dashv p$ & 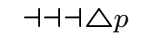 & $\ldots$ & $\dashv \dashv \dashv \triangle^{n} p$ \\
\hline 9 & $\triangle \triangle r$ & $\triangle \triangle \triangle q$ & & & & & $\triangle \triangle \triangle \dashv p$ & $\ldots$ & $\triangle \triangle \triangle \dashv \triangle^{n} p$ \\
\hline 10 & $\dashv \Delta r$ & $\dashv \triangle \Delta q$ & & & & & $\dashv \Delta \triangle \dashv p$ & $\ldots$ & $\dashv \Delta \triangle \dashv \Delta^{n} p$ \\
\hline
\end{tabular}

$$
A_{n}=\langle x \in \Omega \cup S: \operatorname{rank} x \leq n\rangle .
$$

The elements of $\Omega$ of low rank are as given below: 
The next rows follow the same pattern: elements of rank $n$ are determined by writing backwards $n-1$ in base 2 , using the symbols $\Delta=0$ and $\dashv=1$.

Theorem 6.1. We have for all $i \in \mathbb{N}$

$$
\operatorname{Aut}^{i}(\mathfrak{G})=\langle a, b, c, d, x \in \Omega \text { with } \operatorname{rank} x \leq i\rangle .
$$

In particular, $\operatorname{Aut}^{3 \cdot 2^{n-1}}(\mathfrak{G})$ contains $v * \mathfrak{G}$ for all $v \in X^{n}$, since $\operatorname{rank}\left(\dashv^{n} a\right)=3 \cdot 2^{n-1}$.

For all $i \in \mathbb{N}$, we have $\operatorname{Out}\left(\operatorname{Aut}^{i}(\mathfrak{G})\right)=(\mathbb{Z} / 2)^{\omega}$ generated by the $x \in \Omega$ of rank $i+1$.

We have $\operatorname{Aut}^{\alpha}(\mathfrak{G})=\langle\Omega\rangle=\mathfrak{G}_{*}$ for all $\alpha \geq \omega$.

Lemma 6.2. For all $s \in S$, the subgroups $\langle s\rangle^{\mathfrak{G}}$ are all characteristic and distinct.

Proof. The level stabilizer $\operatorname{Stab}_{\mathfrak{G}}(n)$ is characteristic in $\mathfrak{G}$ for all $n$, by Theorem 3.7 The subgroup $\langle s\rangle^{\mathfrak{G}}$ contains $\operatorname{Stab}_{\mathfrak{G}}(4)$, by direct computation [2, Lemma 4.1]. To check that $\langle s\rangle^{\mathfrak{G}}$ is normal in Aut $(\mathfrak{G})$, it is therefore sufficient to check that $\langle s\rangle^{\mathfrak{G}} / \operatorname{Stab}_{\mathfrak{G}}(4)$ is normal in $\operatorname{Aut}(\mathfrak{G}) / \operatorname{Stab}_{\mathfrak{G}}(4)$.

Now Aut $(\mathfrak{G})<W$ by Theorem [3.7 so all computations may be performed in $\bar{W}=W / \operatorname{Stab}_{W}(4)=2^{4} C_{2}$, a 2-Sylow subgroup of $\operatorname{Sym}\left(2^{4}\right)$. The image $\overline{\mathfrak{G}}$ of $\mathfrak{G}$ has order $2^{12}$, and the image of Aut $(\mathfrak{G})$ lies in $\bar{N}=\operatorname{Norm}_{\bar{W}}(\overline{\mathfrak{G}})$, a group of order $2^{13}$. It is then routine to check that $\langle s\rangle^{\mathfrak{G}} / \operatorname{Stab}_{\mathfrak{G}}(4)$ is normal in $\bar{N}$.

These computations were performed using the computer software GAP [12, by expressing $\bar{W}$ as a permutation group on 16 points 5

Define a norm on $\mathfrak{G}_{*}$ by setting

$$
\|g\|=\min \left\{n+\operatorname{rank}\left(\omega_{1}\right)+\cdots+\operatorname{rank}\left(\omega_{n}\right) \mid g=\omega_{1} \ldots \omega_{n}, \omega_{i} \in \Omega \cup S\right\} .
$$

Note that the restriction of this norm to $\mathfrak{G}$ coincides with the norm given by (2), but we shall not need this fact. We have a contraction property on $\mathfrak{G}_{*}$ as in $\mathfrak{G}$ :

Lemma 6.3. For all $g \in \mathfrak{G}_{*}$ and $x \in X$ we have $\|g @ x\| \leq \frac{1}{2}(\|g\|+1)$.

Next define a norm on the set of group homomorphisms $\left\{\phi: \mathfrak{G} \rightarrow \mathfrak{G}_{*}\right\}$ by

$$
\|\phi\|=\sum_{s \in S}\|\phi(s)\| .
$$

Lemma 6.4. Aut $^{\alpha}(\mathfrak{G}) \cong \operatorname{Norm}_{\mathfrak{G}_{*}}^{\alpha}(\mathfrak{G})$ for all ordinals $\alpha \leq \omega$.

Proof. In [21, Theorem 8.5], the authors show that $\mathfrak{G}$ is saturated, with the subgroups $\left(\mho_{2}\right)^{n}(\mathfrak{G})$ fixing $X^{n}$ and acting transitively on each subtree at level $n$. Furthermore, $\mathfrak{G}$ is weakly branch. By Lemma 3.9. the groups $\operatorname{Aut}^{\alpha}(\mathfrak{G})$ are also saturated and weakly branch, so Theorem 3.7 applies, and

$$
\operatorname{Aut}^{\alpha+1}(\mathfrak{G}) \leq \operatorname{Norm}_{W}\left(\operatorname{Aut}^{\alpha}(\mathfrak{G})\right)
$$

for all ordinals $\alpha$.

We proceed by induction on $\alpha$. Take $\phi \in \operatorname{Aut}^{\alpha}(\mathfrak{G})$. First, we may suppose $\phi \in W$ by the above. We construct inductively elements $\phi_{n} \in W$ for all $n \in \mathbb{N}$ by setting $\phi_{0}=\phi$ and decomposing $\phi_{n}=\left(\lambda_{n}, \rho_{n}\right) a^{\varepsilon_{n}}$. We then note that $\lambda_{n}, \rho_{n}: \mathfrak{G} \rightarrow \mathfrak{G}_{*}$

\footnotetext{
${ }^{5}$ Other ad hoc arguments would be preferable for manual computation; for instance, list all quotients of $\mathfrak{G}$ of order 8 and 16 to check that $D$ is characteristic, and that if $B, C$ are not characteristic, then there is an automorphism exchanging them. This last possibility is eliminated by computing the quotients $B / \operatorname{Stab}_{\mathfrak{G}}(3)=D_{8} \times C_{2}$ and $C / \operatorname{Stab}_{\mathfrak{G}}(3)=C_{2}^{4}$.
} 
since $\operatorname{Stab}_{\mathfrak{G}}(1)$ is subdirect in $\mathfrak{G} \times \mathfrak{G}$. We set $\phi_{n+1}$ to be that among $\lambda_{n}, \rho_{n}$ of smallest $\|$-norm, and proceed.

We may assume $\varepsilon_{n}=0$, up to multiplying by an element on $\mathfrak{G}_{*}$. Suppose $\rho_{n}$ is of minimal norm. Then

$$
\begin{aligned}
\left\|\phi_{n+1}\right\| & =\left\|\phi_{n+1}(a)\right\|+\left\|\phi_{n+1}(b)\right\|+\left\|\phi_{n+1}(c)\right\|+\left\|\phi_{n+1}(d)\right\| \\
& =\| \phi_{n}(\text { axa }) @ 2\|+\| \phi_{n}(d) @ 2\|+\| \phi_{n}(b) @ 2\|+\| \phi_{n}(c) @ 2 \| \\
& \leq \frac{1}{2}\left(2\left\|\phi_{n} a\right\|+\left\|\phi_{n} x\right\|+\left\|\phi_{n} b\right\|+\left\|\phi_{n} c\right\|+\left\|\phi_{n} d\right\|+1\right) \\
& <\left\|\phi_{n}\right\|
\end{aligned}
$$

for any $x \in\{b, c\}$; a similar equation holds if $\lambda_{n}$ is of minimal norm. Therefore $\left\|\phi_{n}\right\|=4$ for $n$ large enough, and by Lemma 6.2 we have $\phi_{n}=1$.

Now for $m=n-1, n-2, \ldots, 0$ we have $\left[\dashv \phi_{m}, a\right]=\left(\lambda_{m}^{-1} \rho_{n}, \rho_{m} \lambda_{n}^{-1}\right) \in \operatorname{Aut}^{\alpha}(\mathfrak{G}) \subseteq$ $\mathfrak{G}_{*}$, and since one of $\lambda_{m}, \rho_{m}$ is $\phi_{m+1}$ and therefore belongs to $\mathfrak{G}_{*}$, we obtain that both of them belong to $\mathfrak{G}_{*}$, and therefore $\phi_{m}$ belongs to $\mathfrak{G}_{*}$. We have shown that $\phi_{0} \in \mathfrak{G}_{*}$.

Proof of Theorem 6.1. By Lemma 6.4 the automorphism tower of $\mathfrak{G}$ is contained in $\mathfrak{G}_{*}$. We prove the theorem by showing that the generators of Out $\left(A_{n}\right)$ are precisely the elements of rank $n+1$; for this purpose, we show that

(1) elements of rank $n+1$ normalize $A_{n}$, and

(2) no element of rank $n+2$ or greater normalizes $A_{n}$.

It then follows that $A_{n}=\operatorname{Norm}_{\mathfrak{G}_{*}}^{n}(\mathfrak{G})$.

The following inductive computations show that

$$
\operatorname{rank}[x, y] \leq \max \{\operatorname{rank} x, \operatorname{rank} y\}-1
$$

for $x \in \Omega$ and $y \in \Omega \cup \mathfrak{G}$ :

$$
\begin{aligned}
\operatorname{rank}[\dashv x, \dashv y] & =\operatorname{rank} \dashv[x, y]=2 \operatorname{rank}[x, y] \leq 2(\max \{\operatorname{rank} x, \operatorname{rank} y\}-1) \\
& \leq \max \{\operatorname{rank} \dashv x, \operatorname{rank} \dashv y\}-1 ; \\
\operatorname{rank}[\triangle x, \dashv y] & =\operatorname{rank} \dashv[x, y]=2 \operatorname{rank}[x, y] \leq 2(\max \{\operatorname{rank} x, \operatorname{rank} y\}-1) \\
& \leq \max \{\operatorname{rank} \triangle x, \operatorname{rank} \dashv y\}-1 ; \\
\operatorname{rank}[\triangle x, \triangle y] & =\operatorname{rank} \triangle[x, y] \\
& =2 \operatorname{rank}[x, y]-1 \leq 2(\max \{\operatorname{rank} x, \operatorname{rank} y\}-1)-1 \\
& \leq \max \{\operatorname{rank} \triangle x, \operatorname{rank} \triangle y\}-1 ; \\
\operatorname{rank}[\dashv x, a] & =\operatorname{rank} \triangle x=\operatorname{rank}(\dashv x)-1 ; \\
\operatorname{rank}[\triangle x, a] & =\operatorname{rank} 1=0 ; \\
\operatorname{rank}[\dashv x, b] & =\operatorname{rank} \dashv[x, a]=2 \operatorname{rank}[x, a] \leq 2(\operatorname{rank} x-1) \leq \operatorname{rank}(\dashv x)-1 ; \\
\operatorname{rank}[\triangle x, b] & =\operatorname{rank}(\dashv[x, a])(1,[x, c]) \leq \max \{\operatorname{rank}[x, a], \operatorname{rank}[x, c]\} \\
& \leq \operatorname{rank}(\triangle x)-1 ; \\
{[p, a] } & =(a c)^{8} \in \mathfrak{G}, \operatorname{and} \operatorname{more} \operatorname{generally}[p, s] \in \mathfrak{G} \text { for } s \in S ; \\
\operatorname{rank}[\dashv x, r] & =\operatorname{rank} \dashv[x, a d a d] \leq \operatorname{rank}(\dashv x)-1 ; \\
{[q, s] } & \in \mathfrak{G}\langle p\rangle \text { for } s \in S ; \\
{[r, s] } & \in \mathfrak{G}\langle q\rangle \text { for } s \in S ;
\end{aligned}
$$




$$
[p, q]=1, \quad[p, r]=1, \quad[q, r]=p .
$$

Finally, the tower of $\mathfrak{G}$ stops at level $\omega$ by Theorem 3.15 ,

We note by direct computation that the "branching subgroup" $K$ satisfies the property that $\times^{i}(K)$ is characteristic in Aut ${ }^{2^{i}}(\mathfrak{G})$. It then follows that $\mathfrak{G} \cap \mathfrak{G}^{\alpha}$ has finite index in $\mathfrak{G}$ for all $\alpha \in \operatorname{Aut}^{i}(G)$.

More generally, we have shown for $G \in\{\mathfrak{G}, \ddot{\Gamma}\}$ that $\operatorname{Aut}^{i+1}(G) / \operatorname{Aut}^{i}(G)$ is solvable (actually, of degree $\leq 2$ ) for all $i \in \mathbb{N}$. Therefore, there exists $k \in \mathbb{N}$ such that $\left(\operatorname{Aut}^{i}(G)\right)^{(k)} \leq G$, where $H^{(k)}$ denotes the $k$ th term in the derived series of $H$. Since $G$ is just-infinite and not solvable, it follows that $G \cap G^{\alpha}$ has finite index in both $G$ and $G^{\alpha}$, for all $\alpha \in \operatorname{Aut}^{\omega}(G)$. Hence, Aut ${ }^{\omega}(G)$ is a subgroup of the commensurator of $G$ in $W$.

\section{ACKNOWLEDGEMENT}

The authors express their gratitude to the referee who carefully read this manuscript, and pointed out the relevance of Fodor's lemma to Proposition 1.6.

\section{REFERENCES}

[1] Laurent Bartholdi and Rostislav I. Grigorchuk, On the spectrum of Hecke type operators related to some fractal groups, Trudy Mat. Inst. Steklov. 231, (2000), math.GR/9910102, 5-45. MR1841750 (2002d:37017)

[2] On parabolic subgroups and Hecke algebras of some fractal groups, Serdica Math. J., 28, (2002), math.GR/9911206, 47-90. MR1899368 (2003c:20027)

[3] Laurent Bartholdi, Rostislav I. Grigorchuk and Volodymyr V. Nekrashevych, From fractal groups to fractal sets, Fractals in Graz, (P. Grabner and W. Woess, eds.), Trends in Mathematics, Birkhaüser Verlag, Basel, 2003, pp. 25-118.

[4] Laurent Bartholdi, Lie algebras and growth in branch groups, Pacific J. Math. 218, (2005), no. 1, 241-282.

[5] Yuriı V. Bodnarchuk, The structure of the automorphism group of a nonstandard wreath product of a group, Ukrain. Mat. Zh., 36, (1984), 143-148. MR0742435 (86c:20040)

[6] Martin R. Bridson and Karen Vogtmann, The symmetries of outer space, Duke Math. J., 106, (2001), 391-409. MR 1813435 (2001k:20084)

[7] Andrew M. Brunner and Said N. Sidki, On the automorphism group of the one-rooted binary tree, J. Algebra, 195, (1997), 465-486. MR1469633 (98i:20035)

[8] William Burnside, Theory of groups of finite order, Dover Publications Inc., New York, 1955, 2d ed. MR0069818 (16:1086c)

[9] Joan L. Dyer and Edward Formanek, The automorphism group of a free group is complete, J. London Math. Soc. (2), 11, (1975), 181-190. MR0379683 (52:588)

[10] Jacek Fabrykowski and Narain D. Gupta, On groups with sub-exponential growth functions, J. Indian Math. Soc. (N.S.), 49, (1985), 249-256. MR0942349 (90e:20029)

[11] Ervin Fried and János Kollár, Automorphism groups of fields, Universal Algebra (Esztergom, 1977), North-Holland, Amsterdam, 1982, pp. 293-303. MR0660867 (84i:12020)

[12] GAP - Groups, Algorithms, and Programming, Version 4.3, The GAP Group, 2002, http://www.gap-system.org.

[13] Rostislav I. Grigorchuk, On Burnside's problem on periodic groups, Funktsional. Anal. i Prilozhen., 14, (1980), 53-54, English translation: Functional Anal. Appl. 14 (1980), 41-43. MR0565099 (81m:20045)

[14] - On the Milnor problem of group growth, Dokl. Akad. Nauk SSSR, 271, (1983), 30-33. MR0712546 (85g:20042)

[15] Rostislav I. Grigorchuk and Said N. Sidki, The group of automorphisms of a 3-generated 2-group of intermediate growth, 2003, to appear in International Jounal of Algebra and Computation.

[16] Narain D. Gupta and Said N. Sidki, On the Burnside problem for periodic groups, Math. Z., 182, (1983), 385-388. MR0696534 (84g:20075) 
[17] Joel David Hamkins, Every group has a terminating transfinite automorphism tower, Proc. Amer. Math. Soc., 126, (1998), 3223-3226. MR 487370 (2000e:20057)

[18] Brian Hartley, Simple locally finite groups, Finite and locally finite groups (Istanbul, 1994), Kluwer Acad. Publ., Dordrecht, 1995, pp. 1-44. MR.1362804 (96k:20076)

[19] J. A. Hulse, Automorphism towers of polycyclic groups, J. Algebra, 16, (1970), 347-398. MR0266986 (42:1888)

[20] Winfried Just, Saharon Shelah and Simon Thomas, The automorphism tower problem revisited, Adv. Math., 148, (1999), 243-265. MR.1736959(2001b:20062)

[21] Yaroslav Lavreniuk and Volodymyr V. Nekrashevych, Rigidity of branch groups acting on rooted trees, Geom. Dedicata, 89, (2002), 159-179. MR1890957(2003i:20042)

[22] Paul Lentoudis and Jacques Tits, Sur le groupe des automorphismes de certains produits en couronne, C. R. Acad. Sci. Paris Sér. I Math., 305, (1987), 847-852. MR0925281 (88m:20061)

[23] Jacques Lewin, A finitely presented group whose group of automorphisms is infinitely generated, J. London Math. Soc., 42, (1967), 610-613. MR0222148 (36:5200)

[24] Igor G. Lysionok, A system of defining relations for the Grigorchuk group, Mat. Zametki, 38, (1985), 503-511. MR0819415 (87g:20062)

[25] Darryl McCullough, Finite aspherical complexes with infinitely-generated groups of selfhomotopy-equivalences, Proc. Amer. Math. Soc., 80, (1980), 337-340. MR0577770 (81k:55008)

[26] Rögnvaldur G. Möller, The automorphism groups of regular trees, J. London Math. Soc. (2), 43, (1991), 236-252. MR1111582 (92h:20044)

[27] Peter M. Neumann, On the structure of standard wreath products of groups, Math. Z., 84, (1964), 343-373. MR0188280 (32:5719)

[28] Andrew Rae and James E. Roseblade, Automorphism towers of extremal groups, Math. Z., 117, (1970), 70-75.

[29] Vitaly A. Roman'kov, Automorphisms of groups, Acta Appl. Math., 29, (1992), 241-280. MR0276322 (43:2069)

[30] Claas E. Röver, Abstract commensurators of groups acting on rooted trees, Proceedings of the Conference on Geometric and Combinatorial Group Theory, Part I (Haifa, 200), vol. 94, 2002, pp. 45-61. MR1950873 (2004d:20028)

[31] Matatyahu Rubin, The reconstruction of trees from their automorphism groups, American Mathematical Society, Providence, RI, 1993, ISBN 0-8218-5187-X. MR1240317 (95f:20046)

[32] Said N. Sidki, On a 2-generated infinite 3-group: subgroups and automorphisms, J. Algebra, 110, (1987), 24-55. MR0904180 (89b:20081b)

[33] - On a 2-generated infinite 3-group: the presentation problem, J. Algebra, 110, (1987), 13-23. MR0904179 (89b:20081a)

[34] Regular trees and their automorphisms, 1996, notes for a course, XIV Escola de Álgebra, Rio de Janeiro.

[35] Simon Thomas, The automorphism tower problem, Proc. Amer. Math. Soc., 95, (1985), 166-168. MR0801316 (86k:20028)

[36] — The automorphism tower problem, 2004, to appear; book available electronically at

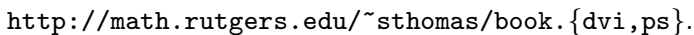

[37] Vladimir Tolstykh, The automorphism tower of a free group, J. London Math. Soc. (2), 61, (2000), 423-440. MR1760692 (2001c:20081)

[38] Helmut Wielandt, Eine Verallgemeinerung der invarianten Untergruppen, Math. Z., 45, (1939), 209-244.

[39] D. V. Znolko, The automorphism groups of regular trees, Mat. Sb. (N.S.), 103(145), (1977), 124-130, 144. MR0444513(56:2864)

École Polytechnique Fédérale, SB/IGAT/MAD, BÂtiment BCH, 1015 Lausanne, SWITZERLAND

E-mail address: laurent.bartholdi@epfl.ch

Universidade de Brasília, Departamento de Matemática, 70.910-900 Brasilia-DF, BRASIL

E-mail address: sidki@mat.unb.br 